\title{
PERFORMING WORK EFFICIENTLY IN THE PRESENCE OF FAULTS*
}

\author{
CYNTHIA DWORK ${ }^{\dagger}$, JOSEPH Y. HALPERN ${ }^{\ddagger}$ AND ORLI WAARTS ${ }^{\S}$
}

\begin{abstract}
We consider a system of $t$ synchronous processes that communicate only by sending messages to one another, and that together must perform $n$ independent units of work. Processes may fail by crashing; we want to guarantee that in every execution of the protocol in which at least one process survives, all $n$ units of work will be performed. We consider three parameters: the number of messages sent, the total number of units of work performed (including multiplicities), and time. We present three protocols for solving the problem. All three are work-optimal, doing $O(n+t)$ work. The first has moderate costs in the remaining two parameters, sending $O(t \sqrt{t})$ messages, and taking $O(n+t)$ time. This protocol can be easily modified to run in any completely asynchronous system equipped with a failure detection mechanism. The second sends only $O(t \log t)$ messages, but its running time is large $\left(O\left(t^{2}(n+t) 2^{n+t}\right)\right)$. The third is essentially time-optimal in the (usual) case in which there are no failures, and its time complexity degrades gracefully as the number of failures increases.
\end{abstract}

Key words. fault-tolerance, work, Byzantine agreement, load balancing, distributed systems

1. Introduction. A fundamental issue in distributed computing is fault-tolerance: guaranteeing that work is performed, despite the presence of failures. For example, in controlling a nuclear reactor it may be crucial for a set of valves to be closed before fuel is added. Thus, the procedure for verifying that the valves are closed must be highly fault-tolerant. If processes never fail then the work of checking that the valves are closed could be distributed according to some load-balancing technique. Since processes may fail, we would like an algorithm that guarantees that the work will be performed as long as at least one process survives. Such an algorithm could be particularly useful in a local area network, where jobs might be distributed among idle workstations. (The idea of running computations on idle nodes is an old one, going back at least to 17. See 12] for one implementation of this approach, and further references.) In this case a "failure" might correspond to a user reclaiming her machine.

The notion of work in this paper is very broad, but is restricted to "idempotent" operations, that is, operations that can be repeated without harm. This is because if a process performs a unit of work and fails before telling a second process of its achievement, then the second process has no choice but to repeat the given unit of work. Examples include verifying a step in a formal proof, evaluating a boolean formula at a particular assignment to the variables, sensing the status of a valve, closing a valve, sending a message, say, to a process outside of the given system, or reading records in a distributed database.

Formally, we assume that we have a synchronous system of $t$ processes that are subject to crash failures, that want to perform $n$ independent units of work. (For

\footnotetext{
* A preliminary version of this work appeared in Proc. 11th ACM Symposium on Principles of Distributed Computing, 1992. This version will appear in SIAM Journal on Computing. During this research, Orli Waarts was at Stanford University and supported by U.S. Army Research Office Grant DAAL-03-91-G-0102, NSF grant CCR-8814921, ONR contract N00014-88-K-0166, and an IBM fellowship.

$\dagger$ IBM Research Division, Almaden Research Center, K53-B2, 650 Harry Road, San Jose, CA 95120-6099. dwork@almaden.ibm.com

$\ddagger$ IBM Research Division, Almaden Research Center, K53-B2, 650 Harry Road, San Jose, CA 95120-6099. halpern@almaden.ibm.com

$\S$ Computer Science Division, U.C. Berkeley, Berkeley, CA 94720. waarts@cs.berkeley.edu
} 
now, we assume that initially there is common knowledge among the $t$ processes about the $n$ units of work to be performed. We return to this point later.) In one time unit a process can compute locally and perform one unit of work and one round of communication (sending and receiving messages). Given that performing a unit of work can be repeated without harm, a trivial solution is obtained by having each process perform every unit of work. In our original example, this would mean that every process checks that every valve is closed. This solution requires no messages, but in the worst case performs $t n$ units of work and runs in $n$ rounds. (Here the worst case is when no process fails.)

Another straightforward solution can be obtained by having only one process performing the work at any time, and checkpointing to each process after completing every unit of work. In this solution, at most $n+t-1$ units of work are ever performed, but the number of messages sent is almost $t n$ in the worst case.

In both these solutions the total amount of effort, defined as work plus messages, is $O(t n)$. If the actual cost of performing a unit of work is comparable to the cost of sending a message, then neither solution is appealing. In this paper we focus on solutions which are work-optimal, up to a constant factor, while keeping the total effort reasonable. Clearly, since a process can fail immediately after performing a unit of work, before reporting that unit to any other process, a work-optimal solution performs $n+t-1$ units of work in the worst case. Thus, we are interested in solutions that perform $O(n+t)$ work.

Let $n^{\prime}=\max (n, t)$. Our first result is an algorithm whose total effort is at most $3 n^{\prime}+9 t \sqrt{t}$. In fact, in the worst case the amount of work performed is at most $3 n^{\prime}$ and the number of messages is at most $9 t \sqrt{t}$, so the form of the bound explains the costs exactly. We then optimize this algorithm to achieve running time of $O(n+t)$ rounds. Note that any solution requires $n$ rounds in the worst case, since if $t-1$ processes are initially faulty then the remaining process must perform all $n$ units of work. In this algorithm the synchrony is used only to detect failures, as usual by detecting the absence of an expected message. Thus, it can be easily modified to work in a completely asynchronous system equipped with a failure detection mechanism.

We then prove that the above algorithm is not message-optimal (among workoptimal algorithms), by constructing a technically challenging work-optimal algorithm that requires only $O(t \log t)$ messages in the worst case. Since $O(n+t)$ is a lower bound on work, and hence on effort, the $O(n+t \log t)$ effort of this algorithm is nearly optimal. The improved message complexity is obtained by a more aggressive use of synchrony. In particular, the absence of a message in this algorithm has two possible meanings: either the potential sender failed or it has insufficient "information" (generally about the history of the execution), and therefore has chosen not to send a message. Due to this use of synchrony, unlike the first algorithm, this low-effort algorithm will not run in the asynchronous model with failure detection. In addition, the efficiency comes at a price in terms of time: in the worst case, the algorithm requires $O\left(t^{2}(n+t) 2^{n+t}\right)$ rounds.

The first two algorithms are very sequential: at all times work is performed by a single active process who uses some checkpointing strategy to inform other processes about the completed work. This forces the algorithms to take at least $n$ steps, even in a failure-free run. To reduce the time we need to increase parallelism. However, intuitively, increasing parallelism while simultaneously minimizing time and remaining work-optimal may increase communication costs, since processes must quickly tell each other about completed work. The third algorithm does exactly this in a fairly 
straightforward way, paying a price in messages in order to decrease best-case time. It is designed to perform time-optimally in the absence of failures, and to have its time complexity degrade gracefully with additional faults. In particular, it takes $n / t+2$ rounds in the failure-free case, and its message cost is $2 t^{2}$; its worst-case message cost is $O\left(f t^{2}\right)$, where $f$ is the actual number of failures in the execution.

There are a number of assumptions in our model that are arguably not realistic. For one, we assume that the $n$ units of work are identical, or, at least, that they all take the same amount of time to perform. In addition, we assume that the total workload is static, and is common knowledge at the beginning of the algorithm. It is not too hard to modify our last algorithm to deal with a more realistic scenario, where work is continually coming in to different sites of the system, and is not initially common knowledge. We remark that a patent has been filed by IBM for such a modified algorithm.

One application of our algorithms is to Byzantine agreement. The idea is that the general tries to inform $t$ processes, and then each of these $t$ processes performs the "work" of ensuring that all processes are informed. In particular, our second algorithm, called Protocol $\mathcal{B}$, gives a Byzantine agreement algorithm for the crash fault model that requires $O(n+t \sqrt{t})$ messages and $O(n)$ time, where $n$ is the number of processes in the system and $t$ is a bound on the number of failures, while our third algorithm gives a Byzantine agreement algorithm that uses $O(n+t \log t)$ messages and exponential time. The best result prior to ours was a nonconstructive algorithm due to Bracha that requires $O(n+t \sqrt{t})$ messages [- 4 . Galil, Mayer, and Yung [11] have recently obtained an algorithm that uses only a linear number of messages. However, the algorithm is incomparable to the agreement algorithm obtained using Protocol $\mathcal{B}$ because it requires a superlinear number of rounds.

Using the observation that our solutions to the work problem yield solutions to Byzantine agreement, we can now return to the assumption that initially there is common knowledge about the work to be performed. Specifically, if even one process knows about this work, then it can act as a general, run Byzantine agreement on the pool of work using one of the three algorithms, and then the actual work is performed by running the same algorithm a second time on the real work. If $n$, the amount of actual work, is $\Omega(t)$, then the overall cost at most doubles when the work is not initially common knowledge.

1.1. Related Work. The idea of doing work in the presence of failures, in a different context, has appeared elsewhere. First, Bridgland and Watro 5 considered a system of $t$ asynchronous processes that together must perform $n$ independent units of work. The processes may fail by crashing and each process can perform at most one unit of work. They provide tight bounds on the number of crash failures that can be tolerated by any solution to the problem.

Clearly, our problem assumes a very different model than the one of [5]. Furthermore, they want a protocol that guarantees that the work will be performed in every execution of the protocol, while we want only a protocol that guarantees that the work will be performed in executions in which at least one process survives. Consequently, their problem is not always solvable and their focus is on finding conditions under which it is solvable. Our problem is always solvable; our focus is on finding efficient solutions.

Another similar but not identical problem was considered by Kanellakis and Shvartsman. In a seminal paper 13 they consider the Write-All problem, in which $n$ processes cooperate to set all $n$ entries of an $n$-element array to the value 1 . They 
provide an efficient solution that tolerates up to $n-1$ faults, and show how to use it to derive robust versions of parallel algorithms for a large class of interesting problems. Their original paper was followed by a number of papers that consider the problem in other shared-memory models (see [1, 6, 14, 15, 16]).

The Write-All problem is, of course, a special case of the type of work we consider. Nevertheless, our framework differs from that of [13] in two important respects, so that their results do not apply to our problem (nor ours to theirs). First, they consider the shared-memory model while we consider the message-passing model. Using the shared-memory model simplifies things considerably for our problem. In this model, there is a straightforward algorithm (that uses shared memory to record what work has been done) with optimal effort $O(n+t)$ (where effort now counts both reading and writing into shared memory, as well as doing work), running in time $O(n t)$. While there are well-known emulators that can translate algorithms from the sharedmemory model to the message-passing model (see [2, 3]), these emulators are not applicable for our problem, because the number of failures they tolerate is less than a majority of the total number of processes, while our problem allows up to $t-1$ failures. Also, these transformations introduce a multiplicative overhead of message complexity that is polynomial in $t$, while one of our goals here is to minimize this term. Second, our complexity measure is inherently different from that of 13. Kanellakis and Shvartsman's complexity measure is the sum, over the rounds during which the algorithm is running, of the number of processes that are not faulty during each round. They call their measure the available processor steps. This measure essentially "charges" for a nonfaulty process at round $r$ whether or not it is actually doing any work (say, reading or writing a cell in shared memory). Our approach is generally not to charge a process in round $r$ if it is not expending any effort (sending a message or performing a unit of work) at that round, since it is free at that round to be working on some other task. [ Of course, the appropriateness of charging or not charging for process idle time will depend very much on the details of the system and the tasks being performed.

Our results have been extended by De Prisco, Mayer, and Yung [8], and by Galil, Mayer, and Yung [11]. De Prisco, Mayer, and Yung [8] consider the problem introduced here, but their goal is to optimize the available processor steps defined by [13], and then the number of messages. They present a message-efficient algorithm that achieves optimality in the available processor steps measure. They also show that when $t \approx n$, any algorithm for performing work in the message-passing model requires at least $n^{2}$ available processor steps. This lower bound can be avoided in sharedmemory models that allow concurrent writes; for example, an $O\left(n \log ^{2} n\right)$ solution is presented in [13]. Galil, Mayer, and Yung [11] employ the results of [8] to obtain a Byzantine agreement algorithm for the crash fault model that requires only a linear number of messages. Roughly speaking, the processors are organized into a tree. The children of the root attempt to solve the problem recursively; the group membership protocol of [8] (called a checkpoint protocol, not to be confused with the checkpoints as defined in our paper) is used to attempt to determine which of the children have failed to complete the recursive step, and the computation is re-organized accordingly.

\footnotetext{
${ }^{1}$ In fact, these emulators are designed for asynchronous systems, and it may be possible to improve their resilience for our synchronous model. Nevertheless, they seem to have an inherent multiplicative overhead in message complexity that is at least linear in $t$.

${ }^{2}$ Inactive processes in our algorithms may need to both receive messages and count the number of rounds that have passed, say from the time they received their last message. We assume that processes can do this while carrying on other tasks.
} 
The Galil, Mayer, and Yung protocol compares to that obtained by using our Protocol $\mathcal{B}$ as follows: 11] requires $O(n)$ messages, while ours requires $O(n \sqrt{n})$; 11 requires $O\left(n^{1+1 / \epsilon}\right)$ rounds of communication while ours requires $O(n)$; finally, 11 . requires messages of length $\Omega\left(n+\log _{2}|V|\right)$, where $V$ is the set of possible agreement values. This appears to be because the protocol requires knowledge of which processors are alive and which processors occupy which parts of the tree. In contrast, our messages are of length $O\left(\log n+\log _{2}|V|\right)$.

2. A Protocol with Effort $O\left(n+t^{3 / 2}\right)$. Our goal in this section is to present a protocol with effort $O(n+t \sqrt{t})$ and running time $O(n+t)$. We begin with a protocol that is somewhat simpler to present and analyze, with effort $O(n+t \sqrt{t})$ and running time $O\left(n t+t^{2}\right)$. This protocol has the additional property of working with minimal change in an asynchronous environment with failure detection.

The main idea of the protocol is to use checkpointing in order to avoid redoing too much work if a process fails. The most naïve approach to checkpointing does not work. To understand why, suppose a process does a checkpoint after each $n / k$ units of work. This means that up to $n / k$ units of work are lost when a process fails. Since up to $t$ processes may fail, this means that $n t / k$ units of work can be lost (and thus must be repeated), which suggests we should take $k \geq t$ if we want to do no more than $O(n)$ units of work altogether. However, since each checkpoint involves $t$ messages, this means that roughly $t k$ messages will be sent. Thus, we must have $k \leq \sqrt{t}$ if we are to use fewer than $t \sqrt{t}$ messages. Roughly speaking, this argument shows that doing checkpoints too infrequently means that there might be a great deal of wasted work, while doing them too often means that there will be a great deal of message overhead. Our protocol avoids these problems by doing full checkpoints to all the processes relatively infrequently - after $n / \sqrt{t}$ units of work - but doing partial checkpoints to only $\sqrt{t}$ processes after every $n / t$ units of work. This turns out to be just the right compromise.

2.1. Description of the Algorithm. For ease of exposition, we assume that $t$ is a perfect square, and that $n$ is divisible by $t$ (so that, in particular, $n>t$ ). We leave to the reader the easy modifications of the protocol when these assumptions do not hold. We assume that the processes are numbered 0 through $t-1$, and that the units of work are numbered 1 through $n$. We divide the processes into $\sqrt{t}$ groups of size $\sqrt{t}$ each, and use the notation $g_{i}$ to denote process $i$ 's group. (Note $g_{i}=\lceil(i+1) / \sqrt{t}\rceil$.) We divide the work into $\sqrt{t}$ chunks, each of size $n / \sqrt{t}$, and subdivide the chunks into $\sqrt{t}$ subchunks of size $n / t$.

The protocol guarantees that at each round, at most one process is active. The active process is the only process performing work. If process $i$ is active, then it knows that processes 0 to $i-1$ have crashed or terminated. Initially, process 0 is active. The algorithm for process 0 is straightforward: Process 0 starts out doing the work, a subchunk at a time. After completing a subchunk $c$, it does a checkpoint to the remaining processes in its group $g_{0}$ (processes 1 to $\sqrt{t}-1$ ); that is, it informs its group that the subchunk of work has been completed by broadcasting to the processes in its group a message of the form $(c)$. (If process 0 crashes in the middle of a broadcast, we assume only that some subset of the processes receive the message.) We call this a partial checkpoint, since the checkpointing is only to the processes in $g_{0}$. (Code for this for module and the whole protocol may be found in Figure 1.) After completing a whole chunk of work - that is, after completing a subchunk $c$ which is a multiple of $\sqrt{t}$-process 0 informs all the processes that subchunk $c$ has been completed, but it informs them one group at a time. After informing a whole group, it checkpoints the 
fact that a group has been informed to its own group (i.e., group 1). Formally, after completing a subchunk $c$ that is a multiple of $\sqrt{t}$, process 0 does a partial checkpoint to its own group, and then for each group $2, \ldots, \sqrt{t}$, process 0 broadcasts to the processes in group $g$ a message of the form $(c, g)$, and then broadcasts to all the processes in its own group a message of the form $(c, g)$. We call this a full checkpoint. Note that in a full checkpoint, there is really a double checkpointing process: we checkpoint both the fact that work has been completed, and (to the processes in $g_{0}$ ) the fact that all processes have been informed that the work has been completed. Process 0 terminates after sending the message $(t, \sqrt{t})$ to process $t-1$, indicating to the last process that the last chunk of work has been completed (unless it crashes before that round).

If process 0 crashes, we want process 1 to become active; if process 1 crashes, we want process 2 to become active, and so on. More generally, if process $j$ discovers that the first $j-1$ processes have crashed, then it becomes active. Once process $j$ becomes active, it continues with essentially the same algorithm as process 0 , except that it does not repeat the work it knows has already been done. We must ensure that the takeover proceeds in a "smooth" manner, so that there is at most one active process at a time.

Process $j$ 's algorithm is as follows. If $j$ does not know that all the work has already been performed and sufficiently long time has passed from the beginning of the execution, then $j$ becomes active. "Sufficiently long" means long enough to ensure that processes $0, \ldots, j-1$ have crashed or terminated. As we show below, we can take "sufficiently long" to be defined by the function $D D(j)=j(n+3 t)$. ("DD" stands for deadline. We remark that this is not an optimal choice for the deadline; we return to this issue later.) Thus, if the round number $r$ is less than $D D(j)$, then $j$ does nothing. Otherwise, if $j$ does not know that the work is completed, it takes over as the active process at round $D D(j)$.

When $j$ takes over as the active process, it essentially follows process 0's algorithm. Suppose the last message $j$ received was of the form $(c, g)$, and this message was received from a process $k$. By the syntax of the message we have that $c$ is a multiple of $\sqrt{t}$ and that $k$ was performing a full checkpoint when it sent the message. If $k \notin g_{j}$ then $g=g_{j}$, since this is the only kind of full checkpoint message that $k$ sends to processes outside its group. Thus, $j$ must inform the rest of its own group that subchunk $c$ was performed, which it does with a Partialcheckpoint $(c)$, and proceeds with the full checkpoint of $c$, beginning with group $g_{i}+1=g+1$.

If $k \in g_{j}$ then $g>g_{j}$; the meaning of $(c, g)$ in this case is that $k$ has told group $g$ that subchunk $c$ has been completed, and is telling its own group, $g_{k}\left(=g_{j}\right)$, about this fact. In this case $j$ first ensures that its own group knows that group $g$ has been informed about subchunk $c$ by broadcasting $(c, g)$ to the remainder of its group, and then proceeds with the full checkpoint beginning with group $g+1$.

If the last message received was of the form $(c)$ then this message was part of a partial checkpoint to $g_{j}$. In this case process $j$ completes the partial checkpoint.

In all three cases, $j$ proceeds with work beginning with subchunk $c+1$ (if such a subchunk exists).

Unless it has already crashed, process $j$ terminates before becoming active if it receives $(t)$ (as part of a partial checkpoint) or $\left(t, g_{j}\right)$ (as part of a full checkpoint). Otherwise, after becoming active at $D D(j)$, it terminates as follows. If $g_{j}=\sqrt{t}$ then $j$ terminates after broadcasting $(t)$ to the remainder of $g_{j}$. If $g_{j}<\sqrt{t}$ then $j$ terminates after completing a call of the form Fullcheckpoint $\left(t, g_{j}+1\right)$. This completes the description of our first protocol. We call this Protocol $\mathcal{A}$; the code appears in 
Main protocol

1. if round number $=D D(j)$ and not received $(t)$ or $\left(t, g_{j}\right)$

2. then DoWork.

\section{DoWork}

1. if the last message received was from $k$ and had the form $(c, g)$

2. then if $k \notin g_{j}$

3. then Partialcheckpoint $(c) ; \quad$ \{see code below

4. $\quad$ else Broadcast $(c, g)$ to processes $j+1, \ldots, g_{j} \sqrt{t}-1$;

5. $\quad$ Fullcheckpoint $(c, g+1) ; \quad$ \{complete a full checkpoint; see code below

6. else let $(c)$ be the last message received;

7. $\quad$ Partialcheckpoint $(c)$;

8. $\quad$ if $c$ is a multiple of $\sqrt{t}$

9. then Fullcheckpoint $\left(c, g_{j}+1\right)$.

10. for $s=c+1$ to $t$ do $\quad$ \{proceed with performing work\}

11. Perform subchunk $s$;

12. Partialcheckpoint $(s)$;

13. $\quad$ if $s$ is a multiple of $\sqrt{t}$;

14. then Fullcheckpoint $\left(s, g_{j}+1\right)$

Partialcheckpoint $(c)$

1. Inform the remainder of group $g_{j}$ that subchunk $c$ has been performed by broadcasting $(c)$ to processes $j+1, \ldots, g_{j} \sqrt{t}-1$

Fullcheckpoint $(c, l)$

1. for $g=l$ to $\sqrt{t}$ do

2. Inform group $g$ that subchunk $c$ has been performed by broadcasting $(c, g)$ to group $g$;

3. Inform the remainder of group $g_{j}$ that group $g$ has been informed about subchunk $c$ by broadcasting $(c, g)$ to processes $j+1, \ldots, g_{j} \sqrt{t}-1$

Fig. 1. Protool $\mathcal{A}$; Code for Process $j$ 
Figure囵.

Notice that we can easily modify this algorithm to run in a completely asynchronous system equipped with an appropriate failure detection mechanism [7]: If a process fails, then the failure detection mechanism must eventually inform all the processes that have not failed of this fact; moreover, the mechanism must be sound, in that it never says that a nonfaulty process has failed. The modification is trivial: rather than waiting until round $D D(j)$ before becoming active, process $j$ waits until it has been informed that processes $1, \ldots, j-1$ crashed or terminated.

2.2. Analysis and Proof of Correctness. We now give a correctness proof for Protocol $\mathcal{A}$. We say a process is retired if it has either crashed or terminated.

Lemma 2.1. A process performs at most $n$ units of work, sends at most $3 t \sqrt{t}$ messages, and runs for less than $n+3 t$ rounds from the time it becomes active to the time it retires.

Proof. It is easy to see that from the time process $i$ becomes active, it performs each unit of work at most once, partial checkpoints each subchunk at most once (and hence performs at most $t$ partial checkpoints), and full checkpoints every chunk at most once (and hence performs at most $\sqrt{t}$ full checkpoints). Each partial checkpoint consists of a broadcast to process $i$ 's group, and hence involves at most $\sqrt{t}$ messages and one round. Thus, process $i$ spends at most $t$ rounds on partial checkpoints, and sends at most $t \sqrt{t}$ messages when performing partial checkpoints. During a full checkpoint, process $i$ broadcasts once to each group other than its own, and broadcasts at most $\sqrt{t}$ times to its own group. Each broadcast involves at most $\sqrt{t}$ messages and one round, and there are $\sqrt{t}$ groups. Thus, process $i$ sends less than $2 t \sqrt{t}$ messages when performing full checkpoints, and takes less than $2 t$ rounds doing so. The required bounds immediately follow.

Recall that $D D(j)=j(n+3 t)$. The following lemma is now immediate from the definition of DD.

Lemma 2.2. Assume process $j$ becomes active at round $r$ of an execution $e_{\mathcal{A}}$ of Protocol $\mathcal{A}$. Then all processes $<j$ have retired before round $r$.

It is sometimes convenient to view a group $g_{i}$ as a whole. Therefore we say that a group is active in the period starting when some process in this group becomes active and ending when the last process of this group retires. Notice that Lemma 2.2 ensures that when $g_{i}$ becomes active, all processes in smaller groups have retired.

Theorem 2.3. In every execution of Protocol $\mathcal{A}$,

(a) at most $3 n$ units of work are performed in total by the processes,

(b) at most $9 t \sqrt{t}$ messages are sent,

(c) by round $n t+3 t^{2}$, all processes have retired.

Proof. Part (c) is immediate from Lemma 2.1 and the definition of $D D$.

We prove parts (a) and (b) simultaneously. To do so, we need a careful way of counting the total number of messages sent and the total amount of work done. A given unit of work may be performed a number of times. If it is performed more than once, say by processes $i_{1}, \ldots, i_{k}$, we say that $i_{2}$ redoes that unit of work of $i_{1}, i_{3}$ redoes the work of $i_{2}$, etc. It is important to note that $i_{3}$ does not redo the work of $i_{1}$ in this case; only that of $i_{2}$. Similarly, we can talk about a message sent during a partial checkpoint of a subchunk or a full checkpoint of a chunk done by $i_{1}$ as being resent by $i_{2}$. In particular, a message $m$ sent by $i_{1}$ as part of a broadcast is resent by $i_{2}$ if $i_{2}$ sends exactly the same message as part of a broadcast (not necessarily to the same set of recipients). For example, if $i_{1}$ sends (c) to the remainder of $g_{i_{1}}$ as part of 
a partial checkpoint, and later $i_{2}$ sends $(c)$ to the remainder of $g_{i_{2}}$, then, whether or not $g_{i_{1}}=g_{i_{2}}$, the messages in the second broadcast are considered to be resendings.

Since the completion of a chunk is followed by a full checkpoint, it is not hard to show that when a new group becomes active, it will redo at most one chunk of work that was already done by previous active groups. It will also redo at most one full checkpoint that was done already on the previous chunk, and $\sqrt{t}$ partial checkpoints (one for each subchunk of work redone). In all, it is easy to see that at most $n / \sqrt{t}$ units of work done by previous groups are redone when a new group becomes active, and $3 t$ messages are resent. Similarly, since the completion of a subchunk is followed by a partial checkpoint, it is not hard to show that when a new process, say $i$, in a group that is already active becomes active, and the last message it received was of the form $(c)$ (i.e., a partial checkpoint of subchunk $c$ ), it will redo at most one subchunk that was already done by previous active process (namely, $c+1$ ), and may possibly resend the messages in two partial checkpoints: the one sent after subchunk $c$, and the one sent after subchunk $c+1$ (if the previous process crashed during the checkpointing of $c+1$ without $i$ receiving the message). If the last message that $i$ received was $(c, g)$ for $g>g_{i}$ (that is, the checkpointing of a checkpoint in the middle of a full checkpoint), then similar arguments show that it may resend $3 \sqrt{t}$ messages: the checkpoint of $(c, g)$ to its own group, the checkpoint $(c, g+1)$ to group $g+1$, and the checkpointing of $(c, g+1)$ to its own group. Thus, the amount of work done by an active group that is redone when a new process in that group becomes active is at most $n / t$, and the number of messages resent is at most $3 \sqrt{t}$.

The maximum amount of unnecessary work done is: (number of groups) $\times($ amount of work redone when a new group becomes active) + (number of processes) $\times$ (amount of work redone when a new process in an already active group becomes active $) \leq \sqrt{t}(n / \sqrt{t})+t(n / t)=2 n$. Similarly, the maximum number of unnecessary messages that may be sent is no more than: (number of groups) $\times$ (number of messages resent when a new group becomes active $)+($ number of processes $) \times($ number of messages resent when a new process in an already active group becomes active) $\leq$ $\sqrt{t}(3 t)+t 3 \sqrt{t}=6 t \sqrt{t}$. Clearly $n$ units of work must be done; by Lemma 2.1, at most $3 t \sqrt{t}$ messages are necessary. Thus, no more than $3 n$ units of work will be done altogether, and no more than $9 t \sqrt{t}$ messages will be sent altogether.

2.3. Improving the Time Complexity. As we have observed, the round complexity of Protocol $\mathcal{A}$ is $n t+3 t^{2}$. We now discuss how the protocol can be modified to give a protocol that has round complexity $O(n+t)$, while not significantly changing the amount of work done or the number of messages sent.

Certainly one obvious hope for improvement is to use a better function than $D D$ for computing when process $i$ should become active. While some improvement is possible by doing this, we can get a round complexity of no better than $O(n \sqrt{t})$ if this is all we do, which is still more than we want. Intuitively, the problem is that if process $j$ gets a message of the form $(c, g)$, then it is possible, as far as $j$ is concerned, that some other process $i<j$ may have received a message of the form $(c+\sqrt{t}, h)$. (Observe that this situation is possible even if $g_{i}=g_{j}$ because if the sender of the message $(c+\sqrt{t}, h)$ crashes at the round it broadcasts this message to $g_{i}$, this message may reach an arbitrary subset of the processes in $g_{i}$.) Process $j$ cannot become active before it is sure that $i$ has retired. To compute how long it must wait before becoming active, it thus needs to compute how long $i$ would wait before becoming active, given that $i$ got a message of the form $(c+\sqrt{t}, h)$. On the other hand, if $i$ did get such a message, then as far as $i$ is concerned, some process $i^{\prime}<i$ may have received a 
message of the form $\left(c+2 \sqrt{t}, h^{\prime}\right)$. Notice that, in this case, process $j$ knows perfectly well that no process received a message of the form $\left(c+2 \sqrt{t}, h^{\prime}\right)$; the problem is that $i$ does not know this, and must take into account this possibility when it computes how long to wait before becoming active. Carrying out a computation based on these arguments gives an algorithm which runs in $O(n \sqrt{t})$ rounds.

On closer inspection, it turns out that the situation described above really causes difficulties only when all processes involved (in the example above, this would be the processes $j, i$, and $i^{\prime}$ ) are in the same group. Thus, in our modified algorithm, called Protocol $\mathcal{B}$, process $j$ computes the time to become active as follows: Suppose that the last message received by process $j$ before round $r$ was received from process $i$ in round $r^{\prime}$. Process $j$ then computes a function $D D_{\mathcal{B}}(j, i)$ with the property that if $r=r^{\prime}+D D_{\mathcal{B}}(j, i)$, then process $j$ knows at round $r$ that all processes in groups $g^{\prime}<g_{j}$ must have retired. Moreover, if $g_{i}=g_{j}$, then $j$ knows at round $r$ that all processes $\leq i$ must have retired. Process $j$ then polls all the lower-numbered processes in its own group not known to it as retired, one by one, to see if they are alive; if not, then $j$ becomes active. If any of them is alive, then the lowest-numbered one that is alive becomes active upon receipt of $j$ 's message. Once a process becomes active, it proceeds just as in Protocol $\mathcal{A}$. This technique turns out to save a great deal of time, while costing relatively little in the way of messages.

In particular, in Protocol $\mathcal{B}$, process 0 follows the same algorithm as in Protocol $\mathcal{A}$. Process $j$ 's algorithm is as follows. Here $j$ receives messages either of the form $(c),(c, g)$ or of the form go ahead. We call the first two types of messages ordinary, to distinguish them from the go ahead messages. Suppose that the last ordinary message received by process $j$ before round $r$ is of the form $(c)$ or $(c, g)$, and this message was received from process $i$ at round $r^{\prime}$. To avoid dealing separately with the special case in which $j$ does not receive any message before it becomes active, we use the convention that process 0 becomes active in round 0 (just before the execution begins) and every process receives from it an ordinary message $(0, g)$ at that round. (These fictitious messages are used only in the analysis and hence will not be taken into account when computing the message complexity of the protocol. Also, if in the actual execution process 0 crashes before ever becoming active then we say that it crashes just after it finishes broadcasting these fictitious messages.) There are now two ways for $j$ to become active at round $r$. The first is if $j$ receives a go ahead message at round $r$ and $c<t$. In this case $j$ becomes active, proceeding just as in Protocol $\mathcal{A}$ when it became active at round $D D(j)$. Alternatively, if $j$ does not receive a message for a sufficiently long time, $j$ becomes active. Intuitively, sufficiently long will ensure that all processes smaller than $j$ have already retired.

To analyze this more formally, we need some definitions. Let PTO be $n / t+2$. ("PTO" stands for process time out.) $P T O-1$ is an upper bound on the number of rounds that can pass before process $j$ in group $g_{j}=g_{i}$ hears from process $i$ if $i$ is active. Let $\bar{\jmath}$ denote $j \bmod \sqrt{t}$. Let $G T O(i)$ be $n / \sqrt{t}+3 \sqrt{t}+(\sqrt{t}-\bar{\imath}-1) P T O+1$. ("GTO" stands for group time out.) $G T O(i)-1$ is an upper bound on the number of rounds that can pass before process $j$ in group $g_{j}>g_{i}$ hears from a process $k$ in $g_{i}$ with $\bar{\imath} \leq \bar{k} \leq \sqrt{t}-1$ if any of these processes is active. Next we define a new deadline function as follows:

$$
D D_{\mathcal{B}}(j, i)= \begin{cases}G T O(i)+\left(g_{j}-g_{i}-1\right) G T O(0) & \text { if } g_{j} \neq g_{i} \\ P T O & \text { otherwise. }\end{cases}
$$

We now define "sufficiently long" in terms of $D D_{\mathcal{B}}$ rather than $D D$. Again taking $r$ to 
be the current round, $r^{\prime}$ to be the last round before $r$ in which $j$ received a message, and $i$ to be the process sending that message, $j$ proceeds as follows: If $r<r^{\prime}+D D_{\mathcal{B}}(j, i)$, then $j$ does nothing. If $c<t$ and $r=r^{\prime}+D D_{B}(j, i), j$ becomes preactive. First consider the case where $g_{i} \neq g_{j}$. Informally, at this point, $j$ knows that all processes from groups $<g_{j}$ have failed. In this case, it sends a go ahead message to each lowernumbered process in its group, starting with the first process in $g_{j}$ up to the $(\bar{\jmath}-1)$ st process in $g_{j}$, and waiting $P T O-1$ rounds between messages to see if it receives a message. (Observe that if the recipient of the go ahead message is alive, the sender receives a message from it within one round after the go ahead mesage was sent; however, for technical reasons the sender of the go ahead messages waits $P T O-1$ rounds between two successive go ahead messages.) If $g_{i}=g_{j}$, process $j$ proceeds similarly to the case where $g_{i} \neq g_{j}$ except that when sending go ahead messages it starts with the $(\bar{\imath}+1)$ st process in $g_{j}$. That is, it sends a go ahead message to each lower-numbered process in its group, starting with the $(\bar{\imath}+1)$ st process in $g_{j}$ up to the $(\bar{\jmath}-1)$ st process in $g_{j}$, and waiting $P T O-1$ rounds between messages to see if it receives a message. If $j$ does not receive any response to its go ahead messages by round $r^{\prime}+D D_{\mathcal{B}}(j, i)+\bar{\jmath} P T O-1$ if $g_{i} \neq g_{j}$, or by round $r^{\prime}+(\bar{\jmath}-\bar{\imath}) P T O-1$ if $g_{i}=g_{j}$, then it becomes active at round $r^{\prime}+D D_{\mathcal{B}}(j, i)+\bar{\jmath} P T O$ (respectively, $\left.r^{\prime}+(\bar{\jmath}-\bar{\imath}) P T O\right)$, proceeding just as it did in Protocol $\mathcal{A}$ at round $D D(j)$. If it does get a message, then $j$ becomes passive again.

Main protocol

1. if just received a go ahead message

2. then DoWork; \{see Figure 1 for details\}

3. else if round number $=r^{\prime}+D D_{\mathcal{B}}(j, i)$

4. then PreactivePhase $\left(i, r^{\prime}\right)$

PreactivePhase $\left(i, r^{\prime}\right)$

1. if $g_{i} \neq g_{j}$

2. then $i^{\prime}:=\left(g_{j}-1\right) \sqrt{t}$;

3. else $i^{\prime}:=i+1$;

4. $\quad r:=$ current round;

5. while not received a message and $i^{\prime}<j$ do

6. if current round $-r \equiv 0(\bmod P T O)$

7. then send go ahead message to process $i^{\prime}$;

$8 . \quad i^{\prime}:=i^{\prime}+1$;

9. if just received a go ahead message or $i^{\prime} \geq j$

10. then DoWork

Fig. 2. Protool $\mathcal{B}$; Code for Process $j$

Note that the construction of the algorithm guarantees that if $r=r^{\prime}+D D_{\mathcal{B}}(j, i)+$ $\bar{\jmath} P T O$, the last ordinary message that $j$ receives in execution $e_{\mathcal{B}}$ prior to round $r$ was sent by $i$, and $g_{i} \neq g_{j}$, then $j$ will become active in $e_{\mathcal{B}}$ at or before round $r$. (It may become active earlier if it receives a go ahead message.) Similarly, if 
$r=r^{\prime}+(\bar{\jmath}-\bar{\imath}) P T O$, the last ordinary message that $j$ receives in execution $e_{\mathcal{B}}$ prior to round $r$ was sent by $i$, and $g_{i}=g_{j}$, then $j$ will become active in $e_{\mathcal{B}}$ at or before round $r$. Define

$$
T T(j, i)= \begin{cases}G T O(i)+\left(g_{j}-g_{i}-1\right) G T O(0)+\bar{\jmath} P T O & \text { if } g_{j} \neq g_{i} \\ (\bar{\jmath}-\bar{\imath}) P T O & \text { otherwise }\end{cases}
$$

(" $T T$ "stands for transition time.) Our observations above show that if $r=r^{\prime}+T T(j, i)$ and the last ordinary message that $j$ receives in execution $e_{\mathcal{B}}$ prior to round $r$ was sent by $i$, then $j$ will become active in $e_{\mathcal{B}}$ at or before round $r$.

Unless it has already crashed, process $j$ terminates before becoming active if it receives $(t)$ (as part of a partial checkpoint) or $\left(t, g_{j}\right)$ (as part of a full checkpoint). Otherwise, after becoming active it terminates as follows. If $g_{j}=\sqrt{t}$ then $j$ terminates after broadcasting $(t)$ to the remainder of $g_{j}$. If $g_{j}<\sqrt{t}$ then $j$ terminates after completing a call of the form Fullcheckpoint $\left(t, g_{j}+1\right)$. This completes the description of Protocol $\mathcal{B}$. The code for Protocol $\mathcal{B}$ appears in Figure 2; it uses the code for the DoWork procedure in Figure 1 .

2.4. Proof of Correctness of Protocol $\mathcal{B}$. In this section we show that the round complexity of Protocol $\mathcal{B}$ is $O(n+t)$, and that neither the amount of work done nor the number of messages sent in Protocol $\mathcal{B}$ is significantly larger than in Protocol $\mathcal{A}$.

Suppose for a moment that in every execution of Protocol $\mathcal{B}$ a process becomes active only after all lower numbered processes have retired. Since when a process becomes active in an execution of Protocol $\mathcal{B}$ it performs essentially the same steps as when it becomes active when it follows Protocol $\mathcal{A}$, a similar proof to the one of Theorem 2.3 will show that the amount of work performed in any execution of Protocol $\mathcal{B}$ is no more than $3 n$ units (which is roughly the maximum amount of work performed in any execution of Protocol $\mathcal{A}$ ), and the number of ordinary messages sent is no more than $9 t \sqrt{t}$ (which is roughly the maximum number of messages sent in any execution of Protocol $\mathcal{A}$ ). Since the number of go ahead messages sent in any execution of Protocol $\mathcal{B}$ is at most $t \sqrt{t}$ (each process sends at most one go ahead message to every other process in its group), it follows immediately that the total amount of effort performed by Protocol $\mathcal{B}$ is not significantly larger than the one performed by Protocol $\mathcal{A}$. Therefore, the main property we need to prove is that in every execution of Protocol $\mathcal{B}$, a process becomes active only after all lower numbered processes have retired.

Our analysis uses what we call activation chains. The round $r$ activation chain of process $i$, denoted $a c(i, r)$, is the sequence of processes $\left\langle i_{m}, \ldots, i_{0}\right\rangle$ such that $i_{0}=i$ and for all $j$, if $i_{j}$ received an ordinary message prior to round $r$, then $i_{j+1}$ is the sender of the last message received by $i_{j}$. (As we show below, it cannot be the case that $i_{j}$ receives ordinary messages from two distinct processes in the same round. Since we have not yet proved this, for now, if $i_{j}$ received ordinary messages from more than one process in the last round in which it received an ordinary message, we take $i_{j+1}$ to be the lowest-numbered process among them.) Notice that our convention that process 0 sent a round 0 message guarantees that $i_{m}=0$. In addition, note that the processes in the activation chain appear in increasing order since a process sends messages only to higher numbered processes.

It is sometimes convenient to view $i$ 's activation chain as a whole and to reason about the effort performed by the chain. We say that process $k$ in $a c(i, r)$ is the current process from the round it becomes active up to (but not including) the round 
at which its successor in the chain becomes active. Note that a process in $a c(i, r)$ is current when it first becomes active. Now let $k, l$ be processes in $a c(i, r)$ such that $l$ immediately succeeds $k$, and assume the last ordinary message $l$ receives from $k$ before $l$ becomes active is sent at round $r^{\prime}$. Clearly, any operation (sending messages or performing a unit of work) done by $k$ after round $r^{\prime}$ is not known by $l$ and hence may be repeated by the chain (that is, may be repeated by some process when it is the current process in the chain). On the other hand, any operation done by $k$ before round $r^{\prime}$ will be known by the processes succeeding it in the chain by the time they become active, and hence will not be repeated by the chain. The operation done by $k$ in round $r^{\prime}$, which is a broadcast to $g_{l}$, will be repeated by $l$ in the first round in which $l$ becomes active. We say that an operation performed by a process $k$ in the chain is useful if it is performed before the round in which the process immediately succeeding $k$ in the chain heard from $k$ for the last time before becoming active (if the process is $i$, then there is no later process in the chain, and hence all operations performed by $i$ are useful). When we refer to an operation performed by a chain ac $(l, r)$, we mean a useful operation performed by some process in that chain. We say that a round is useful for the chain $a c(l, r)$ if the chain performed a useful operation in that round; otherwise we say that the round is useless.

The discussion above shows that the operations performed by a chain proceed in a similar order to the operations performed by a single active process. More precisely, if we consider only useful operations, the processes in the chain perform work units one by one in the natural order and without repetition; each time a subchunk $c$ is completed by the chain, the group of the process that completes this subchunk is informed about this fact exactly once, and if the completed subchunk is a multiple of $\sqrt{t}$, then in addition all groups whose numbers are higher than the group of the process that completed this subchunk are informed that the subchunk is completed one by one in the natural order and without repetition (that is, each such group $g$ receives a message $(c, g)$ exactly once); moreover, each time such a group $g$ is informed, this fact is checkpointed to the group of the informer exactly once. Assume process $i$ is active at some round $r$ with $r \geq r 2 \geq r 1 \geq 1$. Then we denote by $T_{r 1}^{r 2}(i)$ the number of useful rounds for the chain $a c(i, r)$ in interval $[r 1, r 2]$ (that is, in the period from round $r 1$ to round $r 2$ (inclusive)). The discussion above shows the following:

Lemma 2.4. Let $l$ be active at some round $r$ with $r \geq r 2 \geq r 1 \geq 1$. Then

(a) $T_{r 1}^{r 2}(l) \leq n+3 t$,

(b) if $T_{r 1}^{r 2}(l) \geq n / \sqrt{t}+3 \sqrt{t}$, then each process $\geq l$ must have received a message from (a process in) ac $(l, r)$ at some round $r^{\prime}$ such that $r 1 \leq r^{\prime} \leq r 2$.

Proof. Part (a) follows from the fact that in each useful round, the chain either performs work, or checkpoints to some group $g$ the fact that a subchunk $c$ was performed, or checkpoints the fact that group $g$ was informed that chunk $c$ was performed. The discussion above shows that no unit of work is repeated and hence there are at most $n$ useful rounds in which the chain performs work. Similarly, each subchunk is partially checkpointed at most once and hence there are at most $t$ useful rounds in which the chain performs partial checkpoints of subchunks. Also, the completion of a chunk is checkpointed to each group at most once, yielding at most $t$ useful rounds in which such subchunks are checkpointed. Finally, the fact that group $g$ was informed about chunk $c$ is checkpointed at most once, yielding at most $t$ additional useful rounds. Summing the above the claim follows.

Part (b) follows because, as reasoned above, the useful operations done by the chain follow the same order as if they are done by a single active process, and hence 
within $n / \sqrt{t}+3 \sqrt{t}$ rounds the chain must complete a chunk and a full checkpoint.

Now, as we mentioned above, at the core of our proof of correctness is the fact that when a process becomes active, all lower numbered processes have already retired. To prove this, we first prove a lower bound on the number of useful rounds for a given activation chain in a given period. Using this bound, we can show that if some process $i$ receives its last ordinary message before becoming active at round $r 1, i$ becomes active at round $r 2$, and some process $l<i$ has not retired by $r 2$, then process $i$ would have received an ordinary message from some process in $a c(l, r 2)$ between rounds $r 1$ and $r 2$, contradicting our choice of $r 1$.

We now proceed with the formal proofs. We start with a technical lemma.

Lemma 2.5. Let $l>j>k$. Then

(a) $T T(j, k)+T T(l, j)=T T(l, k)$,

(b) if $g_{j}<g_{l}$, then $T T(j, k)+D D_{\mathcal{B}}(l, j)=D D_{\mathcal{B}}(l, k)$.

Proof. The proof is straightforward. We start with Part (a). In the calculations below, we use " $\left(g_{i}=g_{j}\right)$ " to denote the value 1 if $g_{i}=g_{j}$ and 0 otherwise. Similarly, " $\left(g_{i} \neq g_{j}\right)$ " denotes 1 if $g_{i} \neq g_{j}$, and 0 otherwise. Recall that $\bar{\jmath}$ denotes $j \bmod \sqrt{t}$.

$$
\begin{aligned}
T T(j, k)+T T(l, j)= & {\left[G T O(k)+\left(g_{j}-g_{k}-1\right) G T O(0)+\bar{\jmath} P T O\right]\left(g_{j} \neq g_{k}\right) } \\
& +[(\bar{\jmath}-\bar{k}) P T O]\left(g_{j}=g_{k}\right) \\
& +\left[G T O(j)+\left(g_{l}-g_{j}-1\right) G T O(0)+\bar{l} P T O\right]\left(g_{l} \neq g_{j}\right) \\
& +[(\bar{l}-\bar{\jmath}) P T O]\left(g_{l}=g_{j}\right) .
\end{aligned}
$$

If $g_{j}=g_{k}$, then

$$
\begin{aligned}
T T(j, k)+T T(l, j)= & (\bar{\jmath}-\bar{k}) P T O \\
& +\left[G T O(j)+\left(g_{l}-g_{k}-1\right) G T O(0)+\bar{l} P T O\right]\left(g_{l} \neq g_{k}\right) \\
& +[(\bar{l}-\bar{\jmath}) P T O]\left(g_{l}=g_{k}\right) \\
= & {\left[G T O(k)+\left(g_{l}-g_{k}-1\right) G T O(0)+\bar{l} P T O\right]\left(g_{l} \neq g_{k}\right) } \\
& +[(\bar{l}-\bar{k}) P T O]\left(g_{l}=g_{k}\right) \\
= & T T(l, k),
\end{aligned}
$$

and part (a) follows. (In the first equality we replaced $g_{j}$ by $g_{k}$ since in this case they are identical, and the second equality follows because $G T O(j)+(\bar{\jmath}-\bar{k}) P T O=$ $G T O(k)$.

If $g_{j} \neq g_{k}$, then

$$
\begin{aligned}
T T(j, k)+T T(l, j)= & {\left[G T O(k)+\left(g_{j}-g_{k}-1\right) G T O(0)+\bar{\jmath} P T O\right] } \\
& +\left[G T O(j)+\left(g_{l}-g_{j}-1\right) G T O(0)+\bar{l} P T O\right]\left(g_{l} \neq g_{j}\right) \\
& +[(\bar{l}-\bar{\jmath}) P T O]\left(g_{l}=g_{j}\right) \\
= & {\left[G T O(k)+\left(g_{l}-g_{k}-1\right) G T O(0)+\bar{l} P T O\right]\left(g_{l} \neq g_{j}\right) } \\
& +\left[G T O(k)+\left(g_{j}-g_{k}-1\right) G T O(0)+\bar{l} P T O\right]\left(g_{l}=g_{j}\right) \\
= & {\left[G T O(k)+\left(g_{l}-g_{k}-1\right) G T O(0)+\bar{l} P T O\right] } \\
= & T T(l, k),
\end{aligned}
$$

and again part (a) follows. (The second equality follows by a case analysis on whether or not $g_{l}=g_{j}$, using the fact that $G T O(j)+\bar{\jmath} P T O=G T O(0)$ and the fourth equality follows since $g_{j} \neq g_{k}$ and $l>j>k$ implies $g_{l} \neq g_{k}$.) 
The proof of Part (b) is similar. Observe that here by assumption, $g_{l} \neq g_{j}$ and hence also $g_{l} \neq g_{k}$. If $g_{j}=g_{k}$, then

$$
\begin{aligned}
T T(j, k)+D D_{\mathcal{B}}(l, j) & =(\bar{\jmath}-\bar{k}) P T O+\left[G T O(j)+\left(g_{l}-g_{k}-1\right) G T O(0)\right] \\
& =\left[G T O(k)+\left(g_{l}-g_{k}-1\right) G T O(0)\right] \\
& =D D_{\mathcal{B}}(l, k),
\end{aligned}
$$

and part (b) follows.

If $g_{j} \neq g_{k}$, then

$$
\begin{aligned}
T T(j, k)+D D_{\mathcal{B}}(l, j)= & {\left[G T O(k)+\left(g_{j}-g_{k}-1\right) G T O(0)+\bar{\jmath} P T O\right] } \\
& +\left[G T O(j)+\left(g_{l}-g_{j}-1\right) G T O(0)\right] \\
= & {\left[G T O(k)+\left(g_{l}-g_{k}-1\right) G T O(0)\right] } \\
= & D D_{\mathcal{B}}(l, k),
\end{aligned}
$$

and Part (b) follows.

The next lemma establishes a lower bound on the number of useful rounds for an activation chain in a given interval.

Lemma 2.6. Assume $l$ is active at some round $r$ such that $r \geq r 2 \geq r 1 \geq 1$. Assume $p \geq k$ is the current process in ac $(l, r)$ at some round $\leq r 1$. Then $T_{r 1}^{r 2}(l) \geq$ $r 2-r 1-T T(l, k)+1$.

Proof. We first show that if $j$ is in $a c(l, r)$ and becomes active at round $r^{\prime}$ with $r 1 \leq r^{\prime} \leq r 2$, then there are at most $T T(j, k)$ useless rounds in $\left[r 1, r^{\prime}-1\right]$. We proceed by induction on $r^{\prime}$. If $r^{\prime}=r 1$, the result is trivial. If $r^{\prime}>r 1$, then $j$ is $i$ 's successor for some $i$ in the activation chain and $j$ received its last message from $i$ at some round $r^{\prime \prime}$. (There is such an $i$ and such a message since by convention process 0 sent an ordinary message to everybody just before the execution begins.) By definition, we have $r^{\prime} \leq r^{\prime \prime}+T T(j, i)$. If $i=k$, we are done, since no round in $\left[r 1, r^{\prime \prime}-1\right]$ is useless, so there are at most $T T(j, k)=T T(j, i)$ useless rounds in $\left[r 1, r^{\prime}-1\right]$. If $i>k$, then suppose $i$ becomes active at $r^{\prime \prime \prime}$. By the inductive hypothesis, there are at most $T T(i, k)$ useless rounds in $\left[r 1, r^{\prime \prime \prime}-1\right]$. All the rounds in $\left[r^{\prime \prime \prime}, r^{\prime \prime}-1\right]$ are useful. Thus, there are at most $T T(j, i)+T T(i, k)$ useless rounds in $\left[r 1, r^{\prime}-1\right]$. Since $T T(j, i)+T T(i, k)=T T(j, k)$ by Lemma 2.5, the inductive step follows.

Suppose that $l$ becomes active at round $r 3$. By the argument above, there are at most $T T(l, k)$ useless rounds in $[r 1, r 3-1]$. If $r 3>r 2$, it immediately follows that there are at most $T T(l, k)$ useless rounds in $[r 1, r 2]$. On the other hand, if $r 3<r 2$, since $l$ is still active at $r>r 2$, it follows that there are no useless rounds in $[r 3, r 2]$. Hence, we again get that there are at most $T T(l, k)$ useless rounds in $[r 1, r 2]$. The lemma follows.

The next lemma shows that in every execution of Protocol $\mathcal{B}$, by the time a process becomes active, all lower numbered processes have retired.

Lemma 2.7. In every execution of Protocol $\mathcal{B}$,

(a) before the round $r$ in which process $i$ becomes preactive, all processes in groups $<g_{i}$ have retired;

(b) before the round $r$ in which process $i$ becomes active, all processes $<i$ have retired.

Proof. Fix an execution $e_{\mathcal{B}}$ of Protocol $\mathcal{B}$. The proof proceeds by induction on the round $r$. The base case of $r=0$ holds trivially since only process 0 is active then. Assume the claim for $<r$, and we will show it for $r$. If $i=0$, the claim holds trivially. 
Thus, we can assume $i>0$. Suppose that the last ordinary message that $i$ received before round $r$ came from $k$, and was received at round $r 1$. (Note that there must have been such an ordinary message, given our assumption that process 0 sent an ordinary message to all the processes before the execution begins.)

We first prove part (a). Assume, by way of contradiction, that some process $l$ with $g_{l}<g_{i}$ does not retire by round $r$.

Since, by assumption, $r 1$ was the latest round $<r$ at which $i$ received an ordinary message, to complete the proof it is enough to show that if $l$ does not retire before round $r, i$ must have received an ordinary message at some round $r^{\prime \prime}$ with $r 1<r^{\prime \prime}<r$. In fact, we plan to show that $i$ must have received a message in the interval $(r 1, r)$ from some process in $a c(l, r)$. To do this, we plan to use Lemmas 2.4 and 2.6. Notice that both of these lemmas require $l$ to be active. In fact, we can assume without loss of generality that $l$ is active at some round $r 3 \geq r$ of $e_{\mathcal{B}}$, and that $a c(l, r)=a c(l, r 3)$. If not, we can just consider the execution $e_{\mathcal{B}}^{\prime}$ which is identical to $e_{\mathcal{B}}$ up to round $r$, after which all processes other than $l$ crash. It is clear that eventually $l$ becomes active in $e_{\mathcal{B}}$, with the same activation chain it has in round $r$. Moreover, if $i$ receives an ordinary message in the interval $(r 1, r)$ in $e_{\mathcal{B}}^{\prime}$, then it must also receive the same message in $e_{\mathcal{B}}$, since the two executions agree up to round $r$.

Since $k$ becomes active at some round prior to $r$, the inductive hypothesis on part (b) of the lemma implies that all processes $\leq k$ have retired by round $r 1<r$. Thus without loss of generality $l \geq k$. We consider two cases: (i) $k$ is in $a c(l, r)$; (ii) $k$ is not in $a c(l, r)$.

In case (i), since $k$ is in $l$ 's activation chain and is active at round $r 1$, by the inductive hypothesis, it must be the current process in $a c(l, r)$ at round $r 1$. Applying Lemma 2.6 to $a c(l, r 3)=a c(l, r)$, we get

$$
T_{r 1+1}^{r-1}(l) \geq(r-1)-(r 1+1)-T T(l, k)+1 .
$$

By definition, $i$ becomes preactive in round $r=r 1+D D_{\mathcal{B}}(i, k)$, and hence $r-r 1=$ $D D_{\mathcal{B}}(i, k)$. Substituting this into the above inequality we get

$$
T_{r 1+1}^{r-1}(l) \geq D D_{\mathcal{B}}(i, k)-T T(l, k)-1 .
$$

Since $g_{i}>g_{l}$, Lemma 2.5 implies that $D D_{\mathcal{B}}(i, k)-T T(l, k)=D D_{\mathcal{B}}(i, l)$, and substituting this fact in the above inequality we get

$$
\begin{aligned}
T_{r 1+1}^{r-1}(l) & \geq D D_{\mathcal{B}}(i, l)-1 \\
& =G T O(l)+\left(g_{i}-g_{l}-1\right) G T O(0)-1 \\
& =(n / \sqrt{t}+3 \sqrt{t}+(\sqrt{t}-\bar{l}-1) P T O+1)+\left(g_{i}-g_{l}-1\right) G T O(0)-1 \\
& \geq n / \sqrt{t}+3 \sqrt{t} .
\end{aligned}
$$

Thus part (b) of Lemma 2.4 implies that $i$ must have received an ordinary message at some round in the interval $(r 1, r)$, contradicting the assumption that it does not, and the claim follows.

In case (ii), let $k^{\prime}$ be the greatest process $<k$ in l's activation chain, and let $j$ be the smallest process $>k$ in $l$ 's activation chain. Suppose $j$ gets its last message before becoming active from $k^{\prime}$ at round $r 0$. (Note that this means that the last message received by $j$ before becoming active came at $r 0$.) Since the inductive hypothesis on part (b) implies that $k^{\prime}$ must retire before $k$ becomes active, and since $k$ must become active at least one round before it sent a message to $i$ (since by assumption 
$g_{k} \leq g_{l}<g_{i}$ and process $k$ checkpoints to its own group before it sends a message to another group), we have $r 0<r 1-1$. Furthermore, since the processes succeeding $k^{\prime}$ in l's chain are greater than $k$, the same inductive hypothesis implies that these processes can become active only after process $k$ retires, and hence after round $r 1$. Since by definition, any message received by $i$ after round $r 0$ from l's chain must be sent by one of the processes succeeding $k^{\prime}$ in the chain, it follows that if $i$ receives a message from $l$ 's chain after round $r 0$, this message is sent after round $r 1$.

To complete the proof we show that $i$ must have received some message from l's chain at some round in the interval $(r 0, r)$, and hence in the interval $(r 1, r)$, contradicting the assumption that it does not. As argued above, to show this it is enough to show that $T_{r 0+1}^{r-1}(l) \geq n / \sqrt{t}+3 \sqrt{t}$. Applying Lemma 2.6 to $l$ 's activation chain we get

$$
T_{r 0+1}^{r-1}(l) \geq r-1-(r 0+1)-T T\left(l, k^{\prime}\right)+1 .
$$

To bound $T_{r 0+1}^{r-1}(l)$, we need to bound $r-r 0$. To do this we will compute two terms: (a) $r-r 1$; and (b) $r 1-r 0$. The first term is equal to $D D_{\mathcal{B}}(i, k)$ as argued above. To compute the second term, we first show: (1) $g_{j}>g_{k}$, and (2) $g_{k^{\prime}}=g_{k}$.

For (1), clearly $g_{j} \geq g_{k}$, since $j>k$. If $g_{j}=g_{k}, j$ must have received a message from $k$ at round $r 1-1$ before $k$ sent a message to $i$ (since by assumption $g_{k} \leq g_{l}<g_{i}$ and process $k$ checkpoints to its own group just before it sends a message to another group). As we have observed, $r 1-1>r 0$, so this contradicts the assumption that the last message received by $j$ before becoming active came at $r 0$. Thus $g_{j}>g_{k}$.

For (2), clearly $g_{k^{\prime}} \leq g_{k}$. If $g_{k^{\prime}}<g_{k}$, this means that $j$ did not receive a message from a process in $g_{k}$ before becoming active (because if it did, then by the inductive hypothesis on part (b) we have that this message arrives after $k^{\prime}$ retires and hence after round $r 0$ ). But since $g_{j} \leq g_{l}<g_{i}$, and $k$ sent a message to $i$ at $r 1$, some process in $g_{k}$ must have sent a message to $j$ before round $r 1$, and hence before $j$ becomes active. This gives us the desired contradiction.

To complete the proof of case (ii) we use the following claim:

Claim 2.1. Every process $k^{\prime \prime}$ with $k^{\prime}<k^{\prime \prime} \leq k$ that becomes active does so no earlier than round $r 0+\left(\overline{k^{\prime \prime}}-\overline{k^{\prime}}\right) P T O-1$.

Proof. We proceed by induction. Assume $k^{\prime}<k 1 \leq k$ and the claim holds for all $k^{\prime \prime}$ with $k^{\prime}<k^{\prime \prime}<k 1$. We prove it for $k 1$.

We first show that the last ordinary message that any process $\geq k 1$ in $g_{k}$ receives from any process $k 2$ with $k^{\prime} \leq k 2<k 1$ is sent no earlier than round $r 0+(\overline{k 2}-$ $\left.\bar{k}^{\prime}\right) P T O-1$. Observe that since $k^{\prime}$ is in $g_{k}$, so are $k 1$ and $k 2$. If $k 2=k^{\prime}$, the claim trivially follows since $k^{\prime}$ must send a message to its own group at round $r 0-1$ just before it sends a message to $g_{j}>g_{k}$. Otherwise, by the induction hypothesis we have that $k 2$ became active no earlier than round $r 0+\left(\overline{k 2}-\overline{k^{\prime}}\right) P T O-1$, and the claim again follows.

Let $k 2$ be the last process from which $k 1$ receives an ordinary message. Observe that $k^{\prime} \leq k 2<k 1$. (Because, as reasoned above, $k 1$ has received a message from $k^{\prime}$, and hence the message sent from $k 2$ was sent at or after the time the message from $k^{\prime}$; the inductive hypothesis on part (b) therefore implies that $k 2 \geq k^{\prime}$.) It follows from the claim above that the message from $k 2$ was sent no earlier than round $r 0+\left(\bar{k} 2-\overline{k^{\prime}}\right) P T O-1$. In addition, the inductive hypothesis on part (b) implies that $k 1$ becomes active only after $k 2$ retires, and hence only after receiving its message.

Now, assume that $k 1$ does not receive a go ahead message. It then becomes preactive $P T O$ rounds after it receives the last ordinary message from $k 2$ and then $k 1$ 
starts sending go ahead messages to lower numbered processes in its group. Since, by assumption, $k 1$ does not receive a message in response, it becomes active $T T(k 1, k 2)=$ $(\bar{k} 1-k \overline{2}) P T O$ rounds after receiving this last message from $k 2$, and hence no earlier than round $r 0+\left(\bar{k} 1-\overline{k^{\prime}}\right) P T O-1$.

Next assume $k 1$ receives a go ahead message. Let $k 3$ be the process sending this message. Let $k 2$ be the last process from which $k 3$ received an ordinary message before sending the go ahead message to $k 1$. Since $k 3$ sends a go ahead message to $k 1$, it follows that $k 2<k 1$. Just as above, we can show that $k 2 \geq k^{\prime}$, and hence that $k 3$ received the ordinary message from $k 2$ no earlier than round $\geq r 0+\left(\overline{k 2}-\overline{k^{\prime}}\right) P T O-1$. Clearly, $k 3$ sends the go ahead message to $k 1$ no earlier than $(\bar{k} 1-\bar{k} 2) P T O$ rounds after it receives its ordinary message from $k 2$, and the claim follows as above. This completes the proof of the inductive step.

Now, to compute $r 1-r 0$, observe that $r 1$, the round in which $k$ sends a message to $i$, is at least one round after $k$ becomes active (because $g_{i}>g_{k}$ and $k$ first broadcasts to its own group), and hence Claim 2.1 immediately implies that $r 1-r 0 \geq\left(\bar{k}-\bar{k}^{\prime}\right) P T O$. Thus we get that

$$
\begin{aligned}
T_{r 0+1}^{r-1} & \geq(r-r 1)+(r 1-r 0)-T T\left(l, k^{\prime}\right)-1 \\
& \geq D D_{\mathcal{B}}(i, k)+\left(\bar{k}-\overline{k^{\prime}}\right) P T O-T T\left(l, k^{\prime}\right)-1 \\
& =G T O(k)+\left(g_{i}-g_{k}-1\right) G T O(0)+\left(\bar{k}-\overline{k^{\prime}}\right) P T O-T T\left(l, k^{\prime}\right)-1 \\
& =G T O\left(k^{\prime}\right)+\left(g_{i}-g_{k}-1\right) G T O(0)-T T\left(l, k^{\prime}\right)-1 \\
& =G T O\left(k^{\prime}\right)+\left(g_{i}-g_{k^{\prime}}-1\right) G T O(0)-T T\left(l, k^{\prime}\right)-1 \\
& =D D_{\mathcal{B}}\left(i, k^{\prime}\right)-T T\left(l, k^{\prime}\right)-1 .
\end{aligned}
$$

(The fourth inequality follows because $G T O(k)+\left(\bar{k}-\bar{k}^{\prime}\right) P T O=G T O\left(k^{\prime}\right)$, and the fifth inequality follows because $g_{k}=g_{k^{\prime}}$.)

Again, Lemma 2.5 implies that $D D_{\mathcal{B}}\left(i, k^{\prime}\right)-T T\left(l, k^{\prime}\right)=D D_{\mathcal{B}}(i, l)$, and hence

$$
\begin{aligned}
T_{r 0+1}^{r-1} & \geq D D_{\mathcal{B}}(i, l)-1 \\
& =G T O(l)+\left(g_{i}-g_{l}-1\right) G T O(0)-1 \\
& \geq(n / \sqrt{t}+3 \sqrt{t}+(\sqrt{t}-\bar{l}-1) P T O+1)-1 \\
& \geq n / \sqrt{t}+3 \sqrt{t}
\end{aligned}
$$

This completes the proof of the inductive step for part (a).

For part (b), suppose by way of contradiction that $i$ becomes active at round $r$ and process $l<i$ has not retired by round $r$. First assume $i$ does not receive a go ahead message. If $g_{l}<g_{i}$, we get an immediate contradiction using the inductive step for part (a), since $i$ becomes active at or after it becomes preactive. Otherwise, recall that $k$ is the last process from which $i$ receives an ordinary message before becoming active, and this message is received at round $r 1$. If $k>l$, then since $k$ became active before round $r$, the inductive hypothesis on part (b) implies that $l$ must have retired before $k$ became active and hence before round $r$. If $k=l$, then $i$ becomes preactive only after $P T O-1=n / t+1$ additional rounds in which it does not hear from $l$. We claim that $l$ must have retired by that time. Because otherwise, in this period $l$ would have either performed a subchunk and informed its group, or would have checkpointed a subchunk to a group $g \neq g_{l}$ and informed its group about the checkpoint. Since $g_{i}=g_{l}$, in both cases, $i$ must have heard from $l$. Finally, if $l>k$, then before $i$ becomes active it sends a go ahead message to $l$ and waits for 
a message from $l$ for $P T O-1$ additional rounds. Exactly as above, it follows again that since $i$ does not receive any message from $l, l$ must have retired.

Next assume $i$ does get a go ahead message before becoming active. However, the same reasoning as above shows that by the time a process sends a go ahead message to process $i$, all processes $<i$ have retired, and we are done.

Finally we can show:

Theorem 2.8. In every execution of Protocol $\mathcal{B}$,

(a) at most $3 n$ units of work are performed in total by the processes,

(b) at most $10 t \sqrt{t}$ messages are sent,

(c) by round $3 n+8 t$ all processes have retired.

Proof. Parts (a) and (b) were argued in the beginning of Section 2.4 .

For part (c), let $i$ be the last process that is active and consider its activation chain. We want to find the last round $r 2$ in which $i$ is active. It follows from Lemma 2.4 that the maximal number of useful rounds performed by any chain is $n+3 t$. Therefore, applying Lemma 2.6 with $k=0$ we get that

$$
n+3 t \geq T_{1}^{r 2}(i) \geq r 2-1-T T(i, 0)+1 .
$$

Thus

$$
\begin{aligned}
r 2 & \leq n+3 t+T T(i, 0) \\
& \leq n+3 t+T T(t-1,0) \\
& =n+3 t+(\sqrt{t}-1) G T O(0)+(\sqrt{t}-1) P T O \\
& =n+3 t+(\sqrt{t}-1)(n / \sqrt{t}+3 \sqrt{t}+(\sqrt{t}-1)(n / t+2)+1)+(\sqrt{t}-1)(n / t+2) \\
& \leq n+3 t+(\sqrt{t}-1)(n / \sqrt{t}+3 \sqrt{t}+\sqrt{t}(n / t+2)+1) \\
& =n+3 t+(\sqrt{t}-1)(n / \sqrt{t}+3 \sqrt{t}+n / \sqrt{t}+2 \sqrt{t}+1) \\
& \leq n+3 t+\sqrt{t}(2 n / \sqrt{t}+5 \sqrt{t}) \\
& \leq 3 n+8 t .
\end{aligned}
$$

口

3. An Algorithm with Effort $O(n+t \log t)$. In this section we prove that the effort of $O(n+t \sqrt{t})$ obtained by the previous protocols is not optimal, even for work-optimal protocols. We construct another work-optimal algorithm, Protocol $\mathcal{C}$, that requires only $O(n+t \log t)$ messages (and a variant that requires only $O(t \log t)$ messages), yielding a total effort of $O(n+t \log t)$. As is the case with Protocols $\mathcal{A}$ and $\mathcal{B}$, at most one process is active at any given time. However, in Protocol $\mathcal{C}$ it is not the case that there is a predetermined order in which the processes become active. Rather, when an active process fails, we want the process that is currently most knowledgeable to become the new active process. As we shall see, which process is most knowledgeable after an active process $i$ fails depends on how many units of work $i$ performed before failing. As a consequence, there is no obvious variant of Protocol $\mathcal{C}$ that works in the model with asynchronous processes and a failure-detector.

Roughly speaking, Protocol $\mathcal{C}$ strives to "spread out" as uniformly as possible the knowledge of work that has been performed and the processes that have crashed. Thus, each time the active process, say $i$, performs a new unit of work or detects a failure, $i$ tells this to the process $j$ it currently considers least knowledgeable. Then process $j$ becomes as knowledgeable as $i$, so after performing the next unit of work (or detecting another failure), $i$ tells the process it now considers least knowledgeable about this new fact. 
The most naïve implementation of this idea is the following: Process 0 begins by performing unit 1 of work and reporting this to process 1 . It then performs unit 2 and reports units 1 and 2 to process 2 , and so on, telling process $i \bmod t$ about units 1 through $i$. Note that at all times, every process knows about all but at most the last $t$ units of work to be performed.

If process 0 crashes, we want the most knowledgeable alive process - the one that knows about the most units of work that have been done - to become active. (If no process alive knows about any work, then we want the highest numbered alive process to become active.) It can be shown that this can be arranged by setting appropriate deadlines. Moreover, the deadlines are chosen so that at most one process is active at a given time. The most knowledgeable process then continues to perform work, always informing the least knowledgeable process.

The problem with this naïve algorithm is that it requires $O\left(n+t^{2}\right)$ work and $O\left(n+t^{2}\right)$ messages in the worst case. For example, suppose that process 0 performs the first $t-1$ units of work, so that the last process to be informed is process $t-1$, and then crashes. In addition, $t / 2+1, \ldots, t-1$ crash. Eventually process $t / 2$, the most knowledgeable non-retired process, will become active. However, process $t / 2$ has no way of knowing whether process 0 crashed just after informing it about work unit $t / 2$, or process 0 continued to work, informing later processes (who must have crashed, for otherwise they would have become active before process $t / 2$ ). Thus, process $t / 2$ repeats work units $t / 2+1, \ldots, t-1$, again informing (retired) processes $t / 2+1, \ldots t-1$. Suppose process $t / 2$ crashes after performing work unit $t-1$ and informing process $t-1$. Then process $t / 2-1$ becomes active, and again repeats this work. If each process $t / 2-1, t / 2-2, \ldots, 1$, crashes after repeating work units $t / 2+1, \ldots, t-1$, then $O\left(t^{2}\right)$ work is done, and $O\left(t^{2}\right)$ messages are sent. (A slight variant of this example gives a scenario in which $O\left(n+t^{2}\right)$ work is done, and $O\left(n+t^{2}\right)$ messages are sent.)

To prevent this situation, a process performs failure detection before proceeding with the work. The key idea here is that we treat failure detection as another type of work. This allows us to use our algorithm recursively for failure detection. Specifically, fault-detection is accomplished by polling a process and waiting for a response or a timeout. The difficulty encountered by our approach is that, in contrast to the real work, the set of faulty processes is dynamic, so it is not obvious how these processes can be detected without sending (wasteful) polling messages to nonfaulty processes. In fact, in our algorithm we do not attempt to detect all the faulty processes, only enough to ensure that not too much work is wasted by reporting work to faulty processes.

3.1. Description of the Algorithm. For ease of exposition we assume $t$ is a power of 2. Again, the processes are numbered 0 through $t-1$, and the units of work are numbered 1 through $n$. Although our algorithm is recursive in nature, it can more easily be described when the recursion is unfolded. Processing is divided into log $t$ levels, numbered 1 to $\log t$, where level $\log t$ would have been the deepest level of the recursion, had we presented the algorithm recursively. In each level, the processes are partitioned into groups as follows. In level $h, 1 \leq h \leq \log t$, there are $t /\left(2^{\log t-h+1}\right)$ groups of size $2^{\log t-h+1}$. Thus, in level $\log t$, there are $t / 2$ groups of size 2 , in level $\log t-1$ there are $t / 4$ groups of size 4 , and so on, until level 1 , in which there is a single group of size $t$. Let $s_{h}=2^{\log t-h+1}$ denote the size of a group at level $h$. The first group of level $h$ contains processes $0,1, \ldots, s_{h}-1$, the next group contains processes $s_{h}, s_{h}+1, \ldots, 2 s_{h}-1$, and so on. Thus each group of level $h<\log t$ contains two 
1. $h:=\log t$

2. while $h>0$ do

3. DONE := FALSE;

4. while $\neg$ DONE do

5. Send "Are you alive?" to $\operatorname{POINT}_{i}\left[G_{h}^{i}\right]$;

$6 . \quad$ if no response

7. then add $\operatorname{POINT}_{i}\left[G_{h}^{i}\right]$ to $F_{i}$;

$8 . \quad$ if $h \neq \log t$

9. $\quad$ then send ordinary message to point $_{i}\left[G_{h+1}^{i}\right]$;

10. $\operatorname{ROUND}_{i}\left[G_{h+1}^{i}\right]:=$ current round;

11.

12.

13.

14.

15.

16. $\operatorname{POINT}_{i}\left[G_{h+1}^{i}\right]:=\operatorname{successor}\left(\operatorname{POINT}_{i}\left[G_{h+1}^{i}\right]\right)$; if $G_{h}^{i}-F_{i} \neq\{i\}$

then $\operatorname{POINT}_{i}\left[G_{h}^{i}\right]:=\operatorname{successor}\left(\operatorname{POINT}_{i}\left[G_{h}^{i}\right]\right)$;

else DONE $:=$ TRUE;

else (i.e., response received) DONE $:=$ TRUE;

$$
h:=h-1
$$

\{Process level 0 (real work):\}

17. while $\operatorname{POINT}_{i}\left[G_{0}\right] \leq n$ do

18. Perform work unit $\operatorname{POINT}_{i}\left[G_{0}\right]$;

19. Send an ordinary message to $\operatorname{POINT}_{i}\left[G_{1}^{i}\right]$;

20. $\operatorname{ROUND}_{i}\left[G_{1}^{i}\right]:=$ current round;

21. $\operatorname{POINT}_{i}\left[G_{1}^{i}\right]:=\operatorname{successor}\left(\operatorname{POINT}_{i}\left[G_{1}^{i}\right]\right)$;

22. $\operatorname{POINT}_{i}\left[G_{0}\right]:=\operatorname{successor}\left(\operatorname{POINT}_{i}\left[G_{0}\right]\right)$

Fig. 3. Code for Active Process $i$ in Protocol $\mathcal{C}$

groups of level $h+1$. Note that each process $i$ belongs to $\log t$ groups, exactly one on each level. We let $G_{h}^{i}$ denote the level $h$ group of process $i$.

Initially process 0 is active. When process $i$ becomes active, it performs faultdetection in its group at every level, beginning with the highest level and working its way down, leaving level $h$ as soon as it finds a non-faulty process in $G_{h}^{i}$. Once fault-detection has been completed on $G_{1}^{i}$, the set of all processes, process $i$ begins to perform real work. Thus, we sometimes refer to the actual work as $G_{0}$, or level 0 , and the fault-detection on level $h$ as work on level $h$. For each $1 \leq h \leq \log t$, each time it performs a unit of work on $G_{h-1}^{i}$, process $i$ reports that work to some process in $G_{h}^{i}$. (Observe that the above protocol requires at least $n$ messages. However, it will later become clear that modifying this protocol so that when a process performs work on $G_{0}$, it reports only each time it completes $n / t$ units of work, will immediately give a work optimal protocol that requires only $O(t \log t)$ messages.)

A unit of fault-detection is performed by sending a special message "Are you alive?" to one process, and waiting for a reply in the following round. An ordinary message informs a process at some level $h, 1 \leq h \leq \log t$, of a unit of (real or faultdetection) work at level $h-1$. As we shall see, an ordinary message also carries additional information. These two are the only types of messages sent by an active process. As before, a process that has crashed or terminated is said to be retired. An inactive non-retired process only sends responses to "Are you alive?" messages.

Each process $i$ maintains a list $F_{i}$ of processes known by $i$ to be retired. It 
also maintains an array of pointers, POINT $_{i}$, indexed by group name. Intuitively, $\operatorname{POINT}_{i}\left[G_{0}\right]$ is the successor of the last unit of work known by $i$ to have been performed (and therefore this is where $i$ will start doing work when it becomes active). For $h \geq 1, \operatorname{POINT}_{i}\left[G_{h}^{j}\right]$ contains the successor (according to the cyclic order in $G_{h}^{j}$, which we define precisely below) of the last process in $G_{h}^{j}$ known by $i$ to have received an ordinary message from a process in $G_{h}^{j}$ that was performing (real or fault-detection) work on $G_{h-1}^{j}$. We call POINT $_{i}\left[G_{h}^{j}\right]$ process i's pointer into $G_{h}^{j}$. Process $i$ 's moves are governed entirely by the round number, $F_{i}$, and pointers into its own groups (i.e., pointers into groups $G_{h}^{i}$ ). Associated with each pointer $\operatorname{POINT}_{i}[G]$ is a round number, $\operatorname{ROUND}_{i}[G]$, indicating the round at which the last message known to be sent was sent (or, in the case of $G_{0}$, when the last unit of work known to be done was done). Initially, $\operatorname{POINT}_{i}\left[G_{0}\right]=1, \operatorname{POINT}_{i}\left[G_{h}^{j}\right]$ is the lowest-numbered process in $G_{h}^{j}-\{i\}$, and $\operatorname{ROUND}_{i}\left[G_{0}\right]=\operatorname{ROUND}_{i}\left[G_{h}^{j}\right]=0$. We occasionally use $\operatorname{Round}_{i}[G](r)$ to denote the value of $\operatorname{ROUND}_{i}[G]$ at the beginning of round $r$; we similarly use $F_{i}(r)$ and $\operatorname{POINT}_{i}[G](r)$.

The triple $\left(F_{i}\right.$, POINT $_{i}$, ROUND $\left._{i}\right)$ is the view of process $i$. We also define the reduced view of process $i$ to be $\operatorname{POINT}_{i}\left[G_{0}\right]-1+\left|F_{i}\right|$; thus, $i$ 's reduced view is the sum of the number of units of work known by $i$ to be done and the number of processes known by $i$ to be faulty. A process includes its view whenever it sends an ordinary message. When process $i$ receives an ordinary message, it updates its view in light of the new information received. Note that process $i$ may receive information about one of its own groups from a process not in that group. Similarly, it may pass to another process information about a group in which the other process is a member but to which $i$ does not belong.

Let $G_{h}^{i}$ be any group as described above, where the process numbers range from $x$ to $y=x+\left|G_{h}^{i}\right|-1$. There is a natural fixed cyclic order on the group, which we call the cyclic order. Process $i$ sends messages to members of $G_{h}^{i}$ in increasing order. By this we mean according to the cyclic order but skipping itself and all processes in $F_{i}$. Let $j \neq i$ be in $G_{h}^{i}$. Then $j$ 's $i$-successor in $G_{h}^{i}$, is $j$ 's nearest successor in the cyclic ordering that is not in $\{i\} \cup F_{i}$. We omit the $i$ in " $i$-successor," as well as the name of the group in which the successor is to be determined, when these are clear from the context.

When process $i$ first becomes active it searches for other non-retired processes as follows. For each level $h$, starting with $\log t$ and going down to 1 , process $i$ polls group $G_{h}^{i}$, starting with $\operatorname{POINT}_{i}\left[G_{h}^{i}\right]$, by sending an "Are you alive?" message. If no answer is received, it adds this process to $F_{i}$. If $h<\log t$, process $i$ sends an ordinary message reporting this newly detected failure to $\operatorname{POINT}_{i}\left[G_{h+1}^{i}\right]$, sets $\operatorname{POINT}_{i}\left[G_{h+1}^{i}\right]$ to its $i$-successor in $G_{h+1}^{i}$, and sets $\operatorname{ROUND}_{i}\left[G_{h+1}^{i}\right]$ to the current round number. Process $i$ repeats these steps until an answer is received or $G_{h}^{i} \backslash\{i\} \subseteq F_{i}$. It then enters level $h-1$, and repeats the process. Note that if no reply was received, then although the pointer into $G_{h}^{i}$ does not change, the successor in $G_{h}^{i}$ of $\operatorname{POINT}_{i}\left[G_{h}^{i}\right]$ does change, because the successor function takes into account $F_{i}$, which has changed.

Level 0 is handled similarly to levels 1 through $\log t-1$, but the process performs real work instead of polling, and increases the work pointer after performing each unit of work. If $\operatorname{POINT}_{i}\left[G_{0}\right]=n+1$ then process $i$ halts, since in this case all the work has been completed. This completes the description of the behavior of an active process. The code for an active process appears in Figure 3.

At any time in the execution of the algorithm, each inactive non-retired process $i$ has a deadline. We define $D(i, m)$ to be the number of rounds that process $i$ waits 
from the round in which it first obtained reduced view $m$ until it becomes active:

$$
D(i, m)= \begin{cases}K(n+t-m) 2^{n+t-1-m} & \text { if } m \geq 1 \\ K(t-i)(n+t) 2^{n+t-1} & \text { otherwise. }\end{cases}
$$

where $K=5 t+2 \log t$. As we show below (Lemma 3.2), $K$ is an upper bound on the number of rounds that any process needs to wait before first hearing from the active process. (More formally, if $j$ becomes active at round $r$ and is still active $K$ rounds later, then by the beginning of round $r+K$, all processes that are not retired will have received a message from $j$.) All our arguments below work without change if we replace $K$ by any other bound on the number of rounds that a process needs to wait before first hearing from the active process. This observation will be useful later, when we consider a slight modification of Protocol $\mathcal{C}$.

If process $i$ receives no message by the end of $D(i, 0)-1$, then it becomes active at the beginning of round $D(i, 0)$. Otherwise, if at round $r$ it receives a message based on which it obtains a reduced view of $m$, and if it receives no further messages by the end of round $r+D(i, m)-1$, it becomes active at the beginning of round $r+D(i, m)$. This completes the description of the algorithm.

3.2. Analysis and Proof of Correctness. Lemma 3.1. In every execution of Protocol $\mathcal{C}$ in which there are no more than $t-1$ failures, the work is completed.

Proof. By assumption, one of the processes is correct, say $i$. At some point process $i$ will become active, since once every other process has retired process $i$ will not extend its deadline. It is straightforward from inspection of the algorithm that at any time during the execution of the algorithm $\operatorname{POINT}_{i}\left[G_{0}\right]=w$ if and only if the first $w-1$ units of work have been performed, and that when it becomes active, process $i$ performs all units of work from $\operatorname{POINT}_{i}\left[G_{0}\right]$ through $n$.

The next lemma shows that our choice of $K$ has the properties mentioned above.

LEMMA 3.2. If $j$ is active at round $r$, and is not retired by round $r+5 t+2 \log t$, then all processes that are not retired will receive a message from $j$ before the beginning of $r+5 t+2 \log t$.

Proof. It is immediate from the description of the algorithm that all nonretired processes have received a message from $j$ by the time it has performed $t$ units of work (at level $G_{0}$ ) after round $r$. Thus, we compute an upper bound on the time it takes for $j$ to perform $t$ units of work starting at round $r$. In the worst case, $j$ has just become active at the beginning of round $r$, and must do failure detection before reaching level $G_{0}$ and doing work. While doing this failure detection, $j$ sends "are you alive?" messages to at most $t+\log t$ processes (the extra $\log t$ is due to the fact that at each level, it may send one "are you alive?" message to a process that is alive, but crashes later while $j$ is doing failure detection on a larger group). After discovering a failure, process $j$ sends an ordinary message; thus, it sends at most $t$ ordinary messages. Each message sent takes up one round; in addition, process $j$ waits one round for a response after each "are you alive?" message. This means that $j$ spends at most $3 t+2 \log t$ rounds in levels $G_{\log t}^{j}, \ldots, G_{1}^{j}$. Clearly, $j$ spends $\leq 2 t-1$ rounds working at level $G_{0}$ in the course of doing $t$ units of work (since it sends an ordinary message between each unit of work). The required bound follows.

If $i$ received its last ordinary message from $j$ at round $r$, we call other processes that received an ordinary message from $j$ after $i$ did first-generation processes (implicitly, with respect to $i, j$, and $r$ ). If $i$ did not yet receive any ordinary messages, then the first-generation processes (with respect to $i$ and $r$ ) are those that received 
an ordinary message from a process with a number greater than $i$. We define $k$ th generation processes inductively. If we have defined $k$ th generation, then the $(k+1)$ st generation are those processes that receive an ordinary message from a $k$ th generation process. The rank of a process is the highest generation that it is in.

LEMMA 3.3. Let $i$ receive its last ordinary message from $j$ at round $r$, let $m$ be the reduced view of $i$ after receiving this message, and let $\ell$ be a $k$ th rank process with respect to $i, j$, and $r$. Then, after $\ell$ receives its last ordinary message, its reduced view is at least $m+k$.

Proof. The proof is an easy induction on $k$, since when a $k$ th rank process becomes active, it knows about everything its parent knew when it became active, and at least one more piece of work or failure.

We say process $i$ knows more than process $j$ at round $r$ if $F_{i}(r) \supseteq F_{j}(r)$ and for all groups $G, \operatorname{ROUND}_{i}[G](r) \geq \operatorname{ROUND}_{j}[G](r)$. Note that if equality holds everywhere then intuitively the two processes are equally knowledgeable. We first show that our algorithm has the property that for any two inactive non-retired processes, one of them is more knowledgeable than the other, unless they both know nothing; that is, the knowledge of two non-retired processes is never incomparable. This is important so that the "most knowledgeable" process is well-defined. Moreover, the knowledge can be quantified by the reduced view. Process $i$ knows more than inactive process $j$ if and only if the reduced view of $i$ is greater than the reduced view of $j$. Finally, the algorithm also ensures that the active process is at least as knowledgeable as any inactive non-retired process.

LEMMA 3.4. For every round $r$ of the execution the following hold:

(a) If process $i$ received an ordinary message from process $j$ at round $r^{\prime}<r$, and $i$ is inactive and has not retired by the beginning of round $r$, then at the beginning of round $r$, no processes other than $j$ and processes in the $k$ th generation with respect to $i, j$, and $r^{\prime}$, for some $k \geq 1$, know as much as $i$.

(b) Suppose process $i$ received its last ordinary message at round $r^{\prime}$ (if $i$ has received no ordinary messages then $r^{\prime}=0$ ), and $m$ is i's reduced view after receiving this message. If $i$ is not retired at the beginning of round $r=r^{\prime}+$ $D(i, m)$, and it receives no further ordinary messages before the beginning of round $r$, then at the beginning of round $r$ no non-retired process knows more than $i$.

(c) At the beginning of round $r$, there is an asymmetric total order ("knows more than") on the non-zero knowledge of the non-retired processes that did not become active before round $r$, and the active process knows at least as much as the most knowledgeable among these processes. Moreover, for any two nonretired processes $i$ and $j, i$ knows more than $j$ if and only if the reduced view of $i$ is greater than the reduced view of $j$.

(d) At most one process is active in round $r$.

Proof. The proof is by induction on $r$. The base case, $r=1$, is straightforward. Let $r>1$, and assume that all parts of the lemma hold for smaller values of $r$. We prove it for $r$.

For part (a), observe that by the inductive hypothesis, (a) holds at the beginning of round $r-1$. If no process is active in round $r-1$, then no process' knowledge changes, so (a) holds at the beginning of round $r$ as well. If process $j^{\prime}$ is active in round $r-1$, then by part (c) of the inductive hypothesis, $j^{\prime}$ knows at least as much as $i$. Thus, by part (a), it must be the case that $j^{\prime}$ is either $j$ or some process in the $k$ th generation with respect to $i, j$, and $r^{\prime}$, for some $k$ (since, by assumption, $i$ is 
not active at the beginning of round $r$ ). The only process whose knowledge changes during round $r-1$ is one to which $j^{\prime}$ sends an ordinary message. It is immediate from the definition that this process must be in the $k$ th generation with respect to $i$, $j$, and $r$, for some $k$.

For part (b), we must consider two cases: $r^{\prime}>0$ and $r^{\prime}=0$. If $r^{\prime}>0$, let $j$ be the process that wrote to $i$ at $r^{\prime}$. By part (a) we have that only $j$ and processes in generation $k \geq 1$ with respect to $i, j$, and $r^{\prime}$ are as knowledgeable as $i$ at any round in the interval $\left[r^{\prime}, r\right)$. By part (c), these can be the only processes active in this interval. Thus, it suffices to argue that $j$ and all processes of generation $k \geq 1$ with respect to $i, j$, and $r^{\prime}$ are retired by the beginning of round $r$. Since a reduced view is at most $n+t-1$, the highest rank a process could be in is $n+t-1$. We now argue that by the beginning of round $r^{\prime}+D(i, m)>r^{\prime}+(n+t-m) K+D(i, m+1)+\cdots+D(i, n+t-1)$ all processes of ranks 1 through $n+t-1$ have retired. More generally, we argue by induction on $k$ that for every $k$ with $1 \leq k \leq n+t-m-1$, by the beginning of round $s+(k+1) K+D(i, m+1)+\cdots+D(i, m+k)$, every process in ranks 1 to $k$ has retired.

If $k=1$, note that since $i$ received an ordinary message from $j$ at round $r^{\prime}$, by Lemma 3.2, every rank 1 process receives a message from $j$ before round $r^{\prime}+K$. By Lemma 3.3, the reduced view of any such process is at least $m+1$. Since $i$ receives no message from $j$ by round $r^{\prime}+K$, it must be the case that $j$ has retired by round $r^{\prime}+K$. By definition, no rank 1 process can receive any messages at any round in $\left[r^{\prime}+K, r\right)$ (otherwise it would have a rank higher than 1). Thus, any rank 1 process $i^{\prime}$ became active before $r^{\prime}+K+D\left(i^{\prime}, m+1\right)$, so by definition of $K$ and the fact that $D\left(i^{\prime}, m+1\right)=D(i, m+1), i$ would have heard from $i^{\prime}$ before $r^{\prime}+2 K+D(i, m+1)$. It is easy to check that $r^{\prime}+2 K+D(i, m+1)<r^{\prime}+D(i, m)=r$. Since $i$ did not receive any messages by the beginning of round $r, i^{\prime}$ must have retired by then.

In general, consider a rank $k+1$ process $i^{\prime}$, and assume inductively that every rank $k$ or lower process has retired by the beginning of round $r$. By definition of rank, $i^{\prime}$ received an ordinary message from a rank $k$ process, and, since these are all retired by round $r, i^{\prime}$ must have received this message before round $r$. By the inductive hypothesis on $k, i^{\prime}$ must have received its last ordinary message by the beginning of round $r^{\prime}+(k+1) K+D(i, m+1)+\cdots+D(i, m+k)<r$ (again using the fact that $D(i, m)=D\left(i^{\prime}, m\right)$ if $\left.m>0\right)$. By Lemma 3.3, the reduced view of $i^{\prime}$ when it received its last ordinary message before round $r$ was at least $m+k+1$. Thus, it must have become active before round $r^{\prime}+(k+1) K+D(i, m+1)+\cdots+D(i, m+k+1)$, if it became active at all. Since $i$ received no messages from $i^{\prime}$, it follows that $i^{\prime}$ must have retired before round $r^{\prime}+(k+2) K+D(i, m+1)+\cdots+D(i, m+k+1)<r$. This completes the induction on $k$.

If $r^{\prime}=0$ we need the fact that $D(i, 0)>(n+t) K+\max _{j>i}\{D(j, 0)\}+D(i, 1)+$ $\cdots+D(i, n+t-1)$, which follows easily from the definitions. We claim that, for every $k \geq 0$, by round $(k+1) K+\max _{j>i}\{D(j, 0)\}+D(i, 1)+\cdots+D(i, k)$, every process in ranks 1 to $k$ has retired. To see this, note that a rank 0 process $j^{\prime}$ (one with a higher number than $i$ that received no messages) must have become active at round $D\left(j^{\prime}, 0\right)$, and therefore must have retired by round $D\left(j^{\prime}, 0\right)+K$. Thus a level 1 process received its last message by $\max _{j>i}\{D(j, 0)\}+K$. We now proceed as in the case $r^{\prime}>0$.

To prove part (c), observe that the result is immediate from the inductive hypothesis applied to $r-1$ if there is no active process at the beginning of round $r-1$ (for in that case, no process' reduced view changes). Otherwise, suppose that $j$ is active at the beginning of round $r-1$. If $j$ does not send an ordinary message in 
round $r-1$, again the result follows immediately from the inductive hypothesis (since no process' reduced view changes). If $j$ does send an ordinary message to, say, process $i$, it is immediate that $i$ and $j$ know more at the beginning of round $r$ than any other non-retired process, and that $i$ 's reduced view is greater than that of any other non-retired inactive process.

It remains to show part (d). Observe that the result is immediate if no process becomes active at round $r$. Now suppose that process $i$ becomes active at the beginning of round $r$. We must show that no process that was active prior to round $r$ is still active at the beginning of round $r$, and that no process besides $i$ becomes active at round $r$. Let $r^{\prime}$ be the last round in which $i$ received a message (as usual, if $i$ received no messages prior to round $r$, then we take $r^{\prime}=0$ ), and suppose that $m$ was $i$ 's reduced view at round $r^{\prime}$. Then we must have $r=r^{\prime}+D(i, m)$. From part (b), it follows that no non-retired process knows more than $i$ at the beginning of round $r$. From part (c), it follows that any process that was active in the interval $\left[r^{\prime}, r\right)$ must know more than $i$. This shows that all processes that were active before round $r$ must have retired by the beginning of round $r$. Suppose some other process $i^{\prime}$ becomes active at round $r$. We have just shown that $i^{\prime}$ does not know more than $i$. From part (c) it follows therefore that $i^{\prime}$ knows less than $i$. Thus part (b) provides a contradiction to the assumption that $i^{\prime}$ becomes active at round $r$.

LEMMA 3.5. The running time of the algorithm is at most $t K(n+t) 2^{n+t}$ rounds.

Proof. If process $i$ 's reduced view is $m$ and it does not receive a message within $D(i, m)$ steps, then it becomes active. Each message that $i$ receives increases its reduced view. Thus, $i$ becomes active in at most $D(i, 0)+\cdots+D(i, n+t-1)$ rounds. Once it becomes active, arguments similar to those used in Lemma 3.2 show that it retires in at most $2 n+3 t+2 \log t$ rounds. Thus, the running time of the algorithm is at most $D(1,0)+\cdots+D(1, n+t-1)+2 n+3 t+2 \log t \leq t K(n+t) 2^{n+t}$ rounds. $\mathrm{Q}$

The next lemma shows that an active process $i$ does not send messages to retired processes that, because they were more knowledgeable than $i$, should have become active before $i$ did. These messages are avoided because during fault detection $i$ discovers that these processes have retired.

Lemma 3.6. If process $i^{\prime}$ gets an ordinary message at round $r^{\prime}$ from a process operating on group $G_{h-1}^{i^{\prime}}$ and process $i$ is active at the beginning of round $r>r^{\prime}$ then:

(a) if $\operatorname{ROUND}_{i}\left[G_{h}^{i^{\prime}}\right](r) \geq r^{\prime}$, then all processes in the interval $\left[i^{\prime}, \operatorname{POINT}_{i}\left[G_{h}^{i^{\prime}}\right](r)\right)$ in the cyclic order on $G_{h}^{i^{\prime}}$ are either retired by the beginning of round $r$ or receive an ordinary message in the interval $\left[r^{\prime}, \operatorname{ROUND}_{i}\left[G_{h}^{i^{\prime}}\right](r)\right]$ from a process operating on $G_{h-1}^{i^{\prime}}$. (If $i^{\prime}=\operatorname{POINT}_{i}\left[G_{h}^{i^{\prime}}\right](r)$, then all processes in $G_{h}^{i^{\prime}}$ are either retired by the beginning of round $r$ or receive a message in the interval $\left[r^{\prime}, \operatorname{ROUND}_{i}\left[G_{h}^{i^{\prime}}\right](r)\right]$ from a process operating on $G_{h-1}^{i^{\prime}}$.) Moreover, either $i$ 's knowledge at the beginning of round $r$ is greater than $i^{\prime}$ 's knowledge at the end of $r^{\prime}$, or $i^{\prime} \in F_{i}(r)$.

(b) if $\operatorname{ROUND}_{i}\left[G_{h}^{i^{\prime}}\right](r)<r^{\prime}$, then all processes in the interval $\left[\operatorname{POINT}_{i}\left[G_{h}^{i^{\prime}}\right](r), i^{\prime}\right]$ in the cyclic order on $G_{h}^{i^{\prime}}$ are either retired by the beginning of round $r^{\prime}$, or receive a message in the interval $\left(\operatorname{ROUND}_{i}\left[G_{h}^{i^{\prime}}\right](r), r^{\prime}\right]$ from a process operating on $G_{h-1}^{i^{\prime}}$. Moreover, all the processes in this interval are retired by the beginning of round $r$, and if $G_{h}^{i}=G_{h}^{i^{\prime}}$, then all these processes will be in $F_{i}$ by the time $i$ begins to operate on $G_{h-1}^{i}$.

Proof. We proceed by induction on $r$. The case $r=1$ is vacuous. Assume that $r>1$ and the result holds for $r-1$. If $r^{\prime}=r-1$, then it must be the case that $i^{\prime}$ 
received its message from $i, \operatorname{ROUND}_{i}\left[G_{h}^{i^{\prime}}\right](r)=r-1$, and $\operatorname{POINT}_{i}\left[G_{h}^{i^{\prime}}\right]$ is the successor of $i^{\prime}$ in the cyclic order on $G_{h}^{i^{\prime}}$, as computed by $i$ in round $r-1$. It is easy to see that the result follows immediately in this case, because all processes in the interval $\left(i^{\prime}, \operatorname{POINT}_{i}\left[G_{h}^{i^{\prime}}\right]\right)$ must be retired.

Suppose $r^{\prime}<r-1$. If $i$ is also active at round $r-1$, then the result is immediate from the inductive hypothesis unless $\operatorname{ROUND}_{i}\left[G_{h}^{i^{\prime}}\right]$ changes during round $r-1$. The description of the algorithm shows that $\operatorname{ROUND}_{i}\left[G_{h}^{i^{\prime}}\right]$ changes only if $G_{h}^{i}=G_{h}^{i^{\prime}}$ and $i$ is operating on group $G_{h-1}^{i}$, in which case $\operatorname{ROUND}_{i}\left[G_{h}^{i^{\prime}}\right]$ is set to $r-1$ at the end of round $r-1$, and $\operatorname{POINT}_{i}\left[G_{h}^{i^{\prime}}\right](r)$ is the successor of $\operatorname{POINT}_{i}\left[G_{h}^{i^{\prime}}\right](r-1)$ in the cyclic order on $G_{h}^{i^{\prime}}$. In this case it is easy to see that the result follows from the inductive hypothesis; we leave details to the reader.

Thus, we have reduced to the case that $i$ becomes active at round $r$. Let $\operatorname{ROUND}_{i}\left[G_{h}^{i^{\prime}}\right](r)=r^{\prime \prime}$ and let $\operatorname{POINT}_{i}\left[G_{h}^{i^{\prime}}\right](r)=i^{\prime \prime}$. If $r^{\prime \prime} \geq r^{\prime}$, then it must be the case that $i$ received a message from $j$ at some earlier round $s$ such that $\operatorname{POINT}_{j}\left[G_{h}^{i^{\prime}}\right](s)=$ $\operatorname{POINT}_{i}\left[G_{h}^{i^{\prime}}\right](r)$ and $\operatorname{ROUND}_{j}\left[G_{h}^{i^{\prime}}\right](s)=\operatorname{ROUND}_{i}\left[G_{h}^{i^{\prime}}\right](r)$. Since we must have $r^{\prime} \leq r^{\prime \prime}=$ $\operatorname{ROUND}_{j}\left[G_{h}^{i^{\prime}}\right](s) \leq s$, the result now follows from the induction hypothesis (using $j$ and $s$ instead of $i$ and $r$ ).

It remains only to consider the case $r^{\prime \prime}<r^{\prime}$. Let $j^{\prime} \in G_{h}^{i^{\prime}}$ be the process that sent the ordinary message to process $i^{\prime}$ at round $r^{\prime}$, and suppose that $j^{\prime}$ became active at the beginning of round $s^{\prime}$. We claim that we have the following chain of inequalities: $r^{\prime \prime} \leq \operatorname{ROUND}_{j^{\prime}}\left[G_{h}^{i^{\prime}}\right]\left(s^{\prime}\right)<s^{\prime}<r^{\prime}<r$. Every inequality in this chain is immediate from our assumptions except the first one. Suppose that ROUND $j^{\prime}\left[G_{h}^{i^{\prime}}\right]\left(s^{\prime}\right)<r^{\prime \prime}$. From Lemma 3.4, it follows that $\operatorname{RoUND}_{k}\left[G_{h}^{i^{\prime}}\right]\left(s^{\prime}\right)<r^{\prime \prime}$ for all processes $k$ not retired by round $s^{\prime}$. This means that no process not retired at $s^{\prime}$ knows that a message was sent at round $r^{\prime \prime}$. But at round $r>s^{\prime}$, process $i$ knows this fact (since, by assumption $\left.\operatorname{ROUND}_{i}\left[G_{h}^{i^{\prime}}\right](r)=r^{\prime \prime}\right)$. This is impossible. Thus, we must have ROUND $j^{\prime}\left[G_{h}^{i^{\prime}}\right]\left(s^{\prime}\right) \geq r^{\prime \prime}$. Note that $\operatorname{POINT}_{j^{\prime}}\left[G_{h}^{i^{\prime}}\right]\left(r^{\prime}\right)=i^{\prime}$, by assumption. Thus, by the inductive hypothesis, all processes in the cyclic order on $G_{h}^{i^{\prime}}$ in the interval $\left[i^{\prime \prime}, i^{\prime}\right)$ are either retired by the beginning of round $s^{\prime}$ or receive an ordinary message in the interval $\left[r^{\prime \prime}, \operatorname{ROUND}_{j^{\prime}}\left[G_{h}^{i^{\prime}}\right]\left(s^{\prime}\right)\right]$ from a process operating on $G_{h-1}^{i^{\prime}}$. Since we also know that $i^{\prime}$ receives a message at round $r^{\prime}$ from a process operating on $G_{h-1}^{i^{\prime}}$, this proves the first half of part (b). Since, by Lemma 3.4, all processes not retired by round $r$ must be less knowledgeable than $i$ at the beginning of round $r$, it follows from Lemma 3.4 that all the processes in the interval $\left[i^{\prime \prime}, i^{\prime}\right]$ in the cyclic order have in fact retired by round $r$. From the description of the algorithm, it follows that $i$ will detect this fact before it starts operating on $G_{h-1}^{i}$.

Observe that the algorithm treats 'are you alive?' messages as real work. Therefore, we refer to these messages as work unless stated otherwise. On the other hand, the ordinary messages are still referred to as messages.

Using Lemma 3.6, we can show that indeed effort is not wasted:

LEMMA 3.7. At most $\left|G_{h}^{i}\right|+\left|G_{h-1}^{i}\right|$ units of work are done and reported to $G_{h}^{i}$ by group $G_{h}^{i}$ when operating on group $G_{h-1}^{i}$.

Proof. Given $i, h$, and an execution $e$ of Protocol $\mathcal{C}$, we consider the sequence of triples $(x, y, z)$, with one triple in the sequence for every time a process $x \in G_{h}^{i}$ sends an ordinary message reporting a unit of work $y \in G_{h-1}^{i}$ to a process $z \in G_{h}^{i}$, listed in the order that the work was performed. We must show that the length of this sequence is no greater than $\left|G_{h-1}^{i}\right|+\left|G_{h}^{i}\right|$.

We say that a triple $(x, y, z)$ is repeated in this sequence if there is a triple $\left(x^{\prime}, y, z^{\prime}\right)$ 
later in the sequence where the same work unit $y$ is performed. Clearly there are at most $\left|G_{h-1}^{i}\right|$ nonrepeated triples in the sequence, so it suffices to show that there are at most $\left|G_{h}^{i}\right|$ repeated triples. To show this, it suffices to show that the third components of repeated triples (denoting which process was informed about the unit of work) are distinct. Suppose, by way of contradiction, that there are two repeated triples $\left(x_{1}, y_{1}, z_{1}\right)$ and $\left(x_{2}, y_{2}, z_{1}\right)$ with the same third component. Suppose that $x_{1}$ informed $z_{1}$ about $y_{1}$ in round $r^{\prime}$, and $x_{2}$ informed $z_{1}$ about $y_{2}$ in round $r^{\prime \prime}$. Without loss of generality, we can assume that $r^{\prime}<r^{\prime \prime}$. Since $\left(x_{1}, y_{1}, z_{1}\right)$ is a repeated triple, there is a triple $\left(x_{3}, y_{1}, z_{2}\right)$ after $\left(x_{1}, y_{1}, z_{1}\right)$ in the sequence. Let $r_{3}$ be the round in which $x_{3}$ became active, and let $r_{2}$ be the round in which $x_{2}$ became active. Let $s_{j}=\operatorname{ROUND}_{x_{j}}\left[G_{h}^{i}\right]\left(r_{j}\right)$, for $j=2,3$. By Lemma 3.6, if $s_{2} \geq r^{\prime}$, then either $x_{2}$ 's knowledge at the beginning of round $s_{2}$ is greater than $z_{1}$ 's knowledge at the end of $r^{\prime}$, or $z_{1} \in F_{x_{2}}\left(r^{\prime}\right)$, and if $s_{2}<r^{\prime}$, then $z_{1} \in F_{x_{2}}$ before $x_{2}$ starts operating on $G_{i}^{h-1}$. Since $x_{2}$ sends a message to $z_{1}$ while operating on $G_{i}^{h-1}$, it cannot be the case that $z_{1} \in F_{x_{2}}$ before $x_{2}$ starts operating on $G_{i}^{h-1}$, so it must be the case that $s_{2} \geq r^{\prime}$ and $x_{2}$ 's knowledge at the beginning of round $r_{2}$ is greater than $z_{1}$ 's knowledge at the end of round $r^{\prime}$. In particular, this means that $x_{2}$ must know that $x_{1}$ informed $z_{1}$ about $y_{1}$ at the beginning of $r_{2}$.

We next show that every process $x \in G_{i}^{h}$ that is active at some round $r$ between $r^{\prime}$ and $r_{2}$ must know that $x_{1}$ informed $z_{1}$ about $y_{1}$ at the beginning of round $r$. For suppose not. Then, by Lemma 3.6, $z_{1}$ must have retired by the beginning of round $r$. Since, by Lemma 3.4, $x$ is the most knowledgeable process at the beginning of round $r$, it follows that no process that is not retired knows that $z_{1}$ was informed about $y_{1}$. Thus, there is no way that $x_{2}$ could find this out by round $r_{2}$.

It is easy to see that $x_{3}$ does not know that $z_{1}$ was informed about $y_{1}$ (for if it did, it would not repeat the unit of work $\left.y_{1}\right)$. Therefore, $\left(x_{3}, y_{1}, z_{2}\right)$ must come after $\left(x_{2}, y_{2}, z_{1}\right)$ in the sequence. Since POINT $x_{2}\left[G_{h}^{i}\right]\left(r^{\prime \prime}\right)=z_{1}$, and $z_{1}$ received an ordinary message from $x_{1}$ while operating on $G_{h-1}^{i}$ at round $r^{\prime}$, it follows from Lemma 3.6 that between rounds $r^{\prime}$ and $r^{\prime \prime}$, every process in $G_{h}^{i}$ that is not retired must receive an ordinary message. In particular, this means that $x_{3}$ must receive an ordinary message. Since all active processes between round $r^{\prime}$ and $r^{\prime \prime}$ know that $z_{1}$ was informed about $y_{1}$, it follows that $x_{3}$ must know it too by the end of round $r^{\prime \prime}$. But then $x_{3}$ would not redo $y_{1}$, giving us the desired contradiction. $\square$

Theorem 3.8. In every execution of Protocol $\mathcal{C}$,

(a) at most $n+2 t$ units of real work are performed,

(b) at most $n+8 t \log t$ messags are sent,

(c) by round $t(5 t+2 \log t)(n+t) 2^{n+t}$, all processes have retired.

Proof. Lemma 3.7 implies that the amount of real work units that are performed and reported to $G_{1}$ is at most $\left|G_{0}\right|+\left|G_{1}\right|=n+t$. In addition, each of the $t$ processes may perform one unit without reporting it (because it retired immediately afterwards). Summing the two, (a) follows.

For part (b), Lemma 3.7 implies that each $G_{h}^{i}, h>0$, performs at most $\left|G_{h-1}^{i}\right|+$ $\left|G_{h}^{i}\right|$ reported units of works when operating on $G_{h-1}^{i}$. (Here a unit is may be either a real work unit or an 'are you alive?' message.) Let $H=\{(h, i): 1 \leq h \leq \log t, i \equiv 1$ $\left.\left(\bmod 2^{\log (t)+1-h}\right)\right\}$. Notice if we consider groups of the form $G_{h}^{i}$ for $(h, i) \in H$ we count all the groups exactly once. The argument above tells us that the total number of reported units of work is

$$
\sum_{(h, i) \in H}\left(\left|G_{h-1}^{i}\right|+\left|G_{h}^{i}\right|\right) \leq\left|G_{0}\right|+3 \sum_{(h, i) \in H}\left|G_{h}^{i}\right| .
$$


The reason for the factor of 3 is that if $h<\log t$, then $\left|G_{h}^{i}\right|$ occurs three times in the left-hand sum: once when considering the work performed by group $G_{h}^{i}$ operating on $G_{h-1}^{i}$, once when considering the work performed by $G_{h+1}^{i}$ when operating on $G_{h}^{i}$, and once when considering the work performed by $G_{h+1}^{i+h}$ when operating on $G_{h}^{i}$. Clearly, the $\left|G_{0}\right|$ reported units performed on $G_{0}$ result in one message each, and the remaining ones result in two messages each (because then the unit itself is also a message). So the number of messages corresponding to reported units of work is at most

$$
\left|G_{0}\right|+6 \sum_{(h, i) \in H}\left|G_{h}^{i}\right|=n+6 t \log t .
$$

In addition, the unreported units may result in messages. These consist both of 'are you alive?' messages sent by a process but not reported by it due to the fact it crashes or terminates immediately afterwards, and of 'are you alive?' messages that were not reported because the recipient of the 'are you alive?' message responded. Each process in $G_{h}^{i}, h>1$ can perform at most one such unreported unit when operating on $G_{h-1}^{i}$, and hence each group $G_{h}^{i}, h>1$ performs no more than $\left|G_{h}^{i}\right|$ such units. In addition, we have to sum the answers of alive processes in $G_{h-1}^{i}, h>1$ to 'are you alive?' message sent by $G_{h}^{i}$. Again, there are at most $\left|G_{h}^{i}\right|$ such answers. Finally, each process $i$ sends messages to the other process in $G_{\log t}^{i}$ just before it starts operating, which together with the answers sums up to a total of no more than $2 t$ messages. Therefore, the number of messages corresponding to unreported units of work is at most

$$
2 t+\sum_{(i, h) \in H, h>1} 2\left|G_{h}^{i}\right|=2 t \log t .
$$

Summing the messages due to the reported units of work and the messages due to the unreported units of work, part (b) follows.

Part (c) is immediate from Lemma 3.5.

We remark that we can improve the message complexity to $O(t \log t)$ (that is, remove the $n$ term in (b) above) by informing processes in group $G_{1}$ after $n / t$ units of work done at level $G_{0}$, rather than after every unit of work. This does not result in a significant increase in total work, but it does increase the time complexity. The increase in time complexity comes from an increase in $K$ (the upper bound on the number of rounds, from the time the currently active process takes over, that any process needs to wait before first hearing from the active process). Formally, we have

Corollary 3.9. Modifying Protocol $\mathcal{C}$ by informing processes in group $G_{1}$ after $n / t$ units of work done at level $G_{0}$, rather than after every unit of work, yields a protocol that sends $O(t \log t)$ messages, performs $O(n+t)$ work, and terminates within $t(2 n+3 t+2 \log t)(n+t) 2^{n+t}$ rounds.

4. A Time-Optimal Algorithm. All the algorithms we have considered so far are inherently sequential: there is only one process performing work at a time. If processes always have many (other) tasks that they can do, then the fact that all but one process is idle at a given time is not a great problem. On the other hand, time is certainly a critical element in many applications. In this section, we present an algorithm that aims to achieve maximum distribution of the work load among the processes. The algorithm is time-optimal in the typical case where there are no faults, and its performance degrades gracefully in the presence of faults. The basic ideas of this algorithm have been patented [9]. 
The idea of the algorithm is straightforward. We alternate work phases and agreement phases, until all the correct processes are sure that all the work has been done. In the first work phase, process $j$ performs units of work $1+j n / t, \ldots,(j+1) n / t$ (we again assume for simplicity that $n$ is a multiple of $t$ ) in the first $n / t$ rounds. Process $j$ starts the first agreement phase by broadcasting a message to all the other processes saying that it has done its work. In subsequent rounds, process $j$ proceeds much as in Eventual Byzantine Agreement [10]: It broadcasts its current view-what work has been done, and which processes were alive at the end of the work phase, from its point of view. It continues to do so until (a) the set of processes that are currently alive, according to $j$ 's view, is the same in two consecutive rounds, or (b) it receives a message from some process $i$ saying that $i$ is done and containing $i$ 's view. In case (a), it takes as its final view its own view, while in case (b), it takes as its final view the view in $i$ 's message. In all cases, it then broadcasts a message saying it is done, together with its final view of which processes were alive at the end of the work phase and what work remains to be done, and terminates the phase.

Using the by-now standard techniques of [10], we can show that all the correct processes agree on their final view at the time when they terminate the phase, and a correct process is done by round $n / t+f+2$, where $f$ is the number of processes that are faulty during the agreement phase. Finally, all correct processes terminate at most one round after the first correct process terminates. We omit details here.

After process $j$ terminates the first agreement phase, if, according to its final view, $n^{\prime}>0$ more work still needs to be done (perhaps because some process crashed before doing its allocated work) and $t^{\prime} \geq t / 2$ processes are still correct, then it starts the second work phase. It performs $n^{\prime} / t^{\prime}$ units of work, with the work being divided among the correct processes according to their id numbers. $]^{3}$ After the work phase, there is an agreement phase, which is just like the first agreement phase, with one small change. Whereas in the first agreement phase, if process $j$ did not hear from process $i$ during some round, then process $j$ knew $i$ was faulty, in later agreement phases, since $i$ may be behind $j$ by one step, $j$ must allow $i$ one round of grace before declaring it faulty. Similarly, in order to terminate, a process must have two consecutive rounds after the grace round where, its view of the set of currently alive processes is the same, or receive a message from another process saying it is done. We leave it to the reader to check that again, at the end of the phase, all correct processes agree that all the work has been performed, or they agree on their final view, and that every correct process terminates no more than one round after the first correct process terminates.

We continue in this manner, provided no more than half of the processes that were correct at the beginning of a phase fail, until all correct processes are sure that all work has been done. If at any phase more than half the correct processes fail, we revert to another of our algorithms (for example, Protocol $\mathcal{A}$ ). We call this algorithm protocol $\mathcal{D}$; the code appears in Figure 4 . In the code, we use the function grade $_{S}$, where $S$ is a set of nonnegative integers; $\operatorname{grade}_{S}(s)=k$ if there are $k$ elements of $S$ less than $s$.

We now analyze Protocol $\mathcal{D}$. The analysis splits into two cases, depending on whether it is the case that for every phase, no more than half the processes that are correct at the beginning of the phase are discovered to have failed during the phase.

\footnotetext{
3 Since $n^{\prime}$ may not be divisible by $t^{\prime}$, a process might have to do $\left\lceil n^{\prime} / t^{\prime}\right\rceil$ work. We ignore this issue in the discussion, since its impact on complexity is negligible; however, the code takes it into account.
} 
Main protocol

1. $S:=\{1, \ldots, n\} ; \quad\{S$ is the set of outstanding units of work $\}$

2. $T:=\{0, \ldots, t-1\} ; \quad\{T$ is the set of processes known to have been correct at the end of the previous work phase

3. ROUND $:=1 ; \quad$ ROUND keeps track of whether to allow a grace round

4. while $|S|>0$ do

5. $\quad S^{\prime}:=\left\{s \in S: \operatorname{grade}_{T}(j)\lceil|S| /|T|\rceil \leq \operatorname{grade}_{S}(s)<\left(\operatorname{grade}_{T}(j)+1\right)\lceil|S| /|T|\rceil\right\} ;$

6. $\quad$ Perform work in $S^{\prime}$;

7. Wait $\lceil|S| /|T|\rceil-\left|S^{\prime}\right|$ rounds; $\quad$ to make sure all processes spend equally long in this phase

8. $\quad S:=S \backslash S^{\prime} ; \quad$ update outstanding units of work\}

$9 . \quad T^{\prime}:=T$

10. Agree(ROUND); $\quad$ see code below

11. if $\left|T^{\prime}\right|>2|T|$ (i.e. more than half the processes alive at the end of the previous work phase failed by the end of the current work phase)

12. then perform work in $S$ using Protocol $\mathcal{A}$;

13.

14. ROUND $:=0$

Agree(ROUND)

1. DONE $:=$ FALSE;

2. $U:=T ;\{U$ keeps track of processes not known by $j$ to be faulty $\}$

3. $T:=\{j\}$;

4. while $\neg$ DONE do

5. $\quad U_{j}:=U ; \quad$ save old value of $U$ \}

6. $\quad$ Broadcast $(j, S, T$, DONE) to all processes in $U$;

7. $\quad$ for $i \in U_{j}$ do

8. $\quad$ if received $\left(i, S_{i}, T_{i}, \mathrm{DONE}_{i}\right)$ and $\mathrm{DONE}_{i}=\mathrm{FALSE}$

9. then $S:=S \cap S_{i}$;

10. $\quad T:=T \cup T_{i}$;

11. if received $\left(i, S_{i}, T_{i}, \mathrm{DONE}_{i}\right)$ and $\mathrm{DONE}_{i}=\mathrm{TRUE}$

12. then $S:=S_{i}$;

$13 . \quad T:=T_{i}$;

14.

15.

DONE $:=$ TRUE;

if no message received from $i$ and ROUND $\geq 1$

then $U:=U /\{i\}$;

17. $\quad$ if $U=U_{j}$ and ROUND $\geq 1$

18. then DONE $:=$ TRUE;

19. ROUND $:=$ ROUND +1 ;

20. Broadcast $(j, S, T$, DONE) to all processes in $U$

FIG. 4. Protool D; Code for Process $j$ 
A process $p$ is thought to be correct at the beginning of phase $i$ if $i=1$ or $i>1$ and $p$ is in the final view of some process $p^{\prime}$ that decided in the phase $i-1$ agreement protocol. Note that in the latter case $p$ is in the final view of all processes that complete the phase $i-1$ agreement protocol.

THEOREM 4.1. In every execution of Protocol $\mathcal{D}$ in which at most $f$ processes fail,

1. if for each phase, no more than half the processes that are thought to be correct at the beginning of the phase are discovered to have failed by the end of that phase, then

(a) at most $2 n$ units of work are performed,

(b) at most $(4 f+2) t^{2}$ messages are sent,

(c) by round $(f+1) n / t+4 f+2$, all processes have retired.

2. if in some phase more than half the processes that are thought to be correct at the beginning of some phase are discovered to have failed by the end of the phase, then

(a) at most $4 n$ units of work are performed,

(b) at most $(4 f+2) t^{2}+9 t \sqrt{t} /(2 \sqrt{2})$ messages are sent,

(c) by round $(f+1) n / t+4 f+2+n t / 2+3 t^{2} / 4$, all processes have retired.

Proof. For part (1), an easy induction on $k$ shows that by the end of phase $k$, no more than $n / 2^{k}$ units of work remain to be done, and no more than $n+\cdots+n / 2^{k-1}$ units of work have been done. It follows that at most $2 n$ units of work are done altogether. (We remark that there is nothing special about the factor "half" in our requirement that we revert to Protocol $\mathcal{A}$ if more than half the processes that were correct at the beginning of the phase are discovered to have failed during the phase. We could have chosen any factor $\alpha$; a similar proof would show that by the end of phase $k$, at most $\alpha^{k} n$ units of work remain to be done, and no more than $n+\cdots+\alpha^{k-1} n$ units of work have been done, so that no more than $n /(1-\alpha)$ units of work are done altogether. However, it follows from results of [8] that if we allow an arbitrary fraction of the processes to fail at every step, and do not revert to Protocol $\mathcal{A}$, it is possible to construct an execution where $f$ processes fail and $\Omega(n \log (f) / \log \log (f))$ units of work are done altogether. Indeed, it follows from the arguments in [B] that this result is tight; there is a matching upper bound.) Since each nonfaulty process broadcasts to all the other nonfaulty processes in each round of an agreement phase, at most $t^{2}$ messages are sent in each such round. If $f_{k}$ is the number of failures discovered during the $k$ th agreement phase, then the first agreement phase lasts at most $f_{1}+2$ rounds, while for $k>1$, the $k$ th agreement phase lasts at most $f_{k}+3$ rounds, because of the grace round. Thus, altogether, the agreement phases last at most $f+3 a-1$ rounds, where $a$ is the number of agreement phases. Since $a \leq f+1$, the agreement phases last at most $4 f+2$ rounds, and at most $(4 f+2) t^{2}$ messages are sent. Finally, to compute an upper bound on the total number of rounds, it remains only to compute how many rounds are required to do the work (since we know the agreement phases last altogether at most $4 f+2$ rounds). Recall that at the end of phase $k$, at most $n / 2^{k}$ units of work need to be done. Since no more than half the processes fail during any phase, at least $t / 2^{k}$ processes are nonfaulty. Thus, at most $\left(n / 2^{k}\right) /\left(t / 2^{k}\right)=n / t$ rounds are spent during each work phase doing work. Since there are at most $f+1$ work phases, this gives the required bound on the total number of rounds.

For part (2), first observe that if we revert to Protocol $\mathcal{A}$ at the end of phase $k$, then by our earlier observations it is known to the remaining processes that no more than $n / 2^{k-1}$ units of work remain to be done, and no more than $\left(2-1 / 2^{k-1}\right) n$ 
units of work have been done. It is also easy to see that at least $t / 2$ processes are discovered as faulty. Moreover, by the bounds in part $(1)$, at most $(4 f+2) t^{2}$ messages have been sent and $(f+1) n / t+4 f+2$ rounds have elapsed. Now applying Theorem 2.3, we see that at most $3 n / 2^{k-1}$ work is performed by protocol $\mathcal{A}$, no more than $9(t / 2) \sqrt{t / 2}=9 t \sqrt{t} /(2 \sqrt{2})$ messages are sent, and $n t / 2^{k}+3 t^{2} / 4$ rounds are required. By taking $k=1$ (the worst case), we get the bounds claimed in the statement of the theorem.

While the worst-case message complexity of this algorithm is significantly worse than that of our other algorithms, the time complexity is better (at least, if less than half the correct processes fail in each phase). More importantly, the situation is particularly good if no process fails or one process fails. If no process fails, then $n$ units of work are done, the algorithm takes $n / t+2$ rounds, and $2 t^{2}$ messages are sent. If one process fails, then we leave it to the reader to check that the algorithm requires at most $n / t+\lceil n /(t(t-1))\rceil+6$ rounds, has message complexity at most $5 t^{2}$, and at most $n+n / t$ units of work are done.

As we mentioned in the introduction, it is easy to modify this algorithm to deal with a somewhat more realistic setting, where work is continually coming in to the system. Essentially, the idea is to run Eventual Byzantine Agreement periodically (where the length of the period depends on the size of the work load, and other features of the system). We omit further details here.

We can also cut down the message complexity in the case of no failures to $2(t-1)$, rather than $2 t^{2}$, while still keeping the same work and time complexity. Instead of messages being broadcast during the agreement phase, they are all sent to a central coordinator, who broadcasts the results. If there are no failures, the agreement phase lasts 2 rounds, just as before. Dealing with failures is somewhat subtle if we do this though, so we do not analyze this approach carefully here.

5. Application to Byzantine Agreement. One application of our algorithms is to Byzantine agreement. A Byzantine agreement protocol provides a means for $n$ processes, at most $t$ of which may be faulty, to agree on a value broadcast by a distinguished process called the general, in such a way that all nonfaulty processes decide on the same value and, when the general is nonfaulty, they decide on the value the general sent. As in the rest of the paper, we restrict ourselves here to crash failures.

Consider the following Byzantine agreement algorithm. The algorithm proceeds in two stages: first, the general broadcasts its value to processes 0 to $t$; and then, these $t+1$ processes employ one of our sequential algorithms to perform the "work" of informing processes 0 to $n-1$ about the general's value. So, performing one unit of work here means sending a message of the form "the general's value is $x$." To distinguish processes $0, \ldots, t$ from the others, we refer to them as the senders. A few more details are necessary to complete the description of the algorithm. First, throughout the algorithm, each process has a value for the general. Initially, the value is 0 . If a process receives a message informing it about a value for the general different from its current value, it adopts the new value. Second, if the chosen work protocol is $\mathcal{C}$, then we modify it slightly so that each of its messages contains, in addition to its usual original contents, the current value the sender has for the general. Finally, at a predetermined time by which the underlying work protocol is guaranteed to have terminated, each process decides on its current value for the general.

Observe that processes $0, \ldots, t$ play two roles in the second stage of the Byzantine agreement algorithm: they both report the value of the general (as they do work) and 
are informed of this value (as work is performed on them by other senders).

We now prove the correctness of our Byzantine agreement algorithm. Obviously, if the general is correct, all processes will decide on its value. Since at least one of the $t+1$ senders is nonfaulty, it must be the case that a value is reported by a sender to every nonfaulty process. To show agreement, it suffices to show that there is no time at which two nonfaulty processes that have had a value reported to them by a sender have different (current) values. This, in turn, follows from the fact that if an active sender $p$ reports a value $v$, and the sender that was active just before $p$ reported $\bar{v}$, then at the time $p$ becomes active, no value was reported to any nonfaulty process. Assume otherwise. Let $p$ be the first active sender that violates this claim. Then $p$ reports $v$ for the general and the previous sender reported $\bar{v}$. Let $q$ be the first sender that was active before $p$ and reported $\bar{v}$; by construction, all senders that were active after $q$ but before $p$ reported $\bar{v}$.

By assumption, when $q$ becomes active, no value was reported to any process that has not yet crashed. The choice of $q$ guarantees that the only value that is reported from the time that $q$ becomes active to the time that $p$ becomes active is $\bar{v}$. It cannot be the case that a value was reported to $p$ during this time, for then $p$ 's value when it becomes active would be $\bar{v}$, not $v$. In the case of Protocols $\mathcal{A}$ and $\mathcal{B}$, since work is done in increasing order of process number, it follows that no value is reported to any process with a higher number than $p$. (We remark that it is important here that a value is not included as part of the checkpoint messages in Protocols $\mathcal{A}$ and $\mathcal{B}$. Since checkpoint messages are broadcast, if a value were included, it is possible that a process that was active before $p$ crashed while checkpointing to $p$; in this case, $p$ may not have heard the value $\bar{v}$, and some process with a higher number than $p$ may have heard it.) Moreover, the proof of correctness of Protocols $\mathcal{A}$ and $\mathcal{B}$ shows that all processes with a lower number than $p$ must have crashed before $p$ became active. Thus, it follows that no value was reported to any nonfaulty process at the time $p$ became active. In the case of Protocol $\mathcal{C}$, when $p$ becomes active it is the most knowledgeable nonretired (and hence nonfaulty) process. Since for Protocol $\mathcal{C}$ we assume that the checkpointing messages include the value that was sent, no value can have been sent to any process that has not crashed.

Using Protocol $\mathcal{C}$, we get a Byzantine Agreement protocol for crash failures that uses $O(n+t \log t)$ messages in the worst case, thus improving over Bracha's bound of $O(n+t \sqrt{t})$ [4. Using $\mathcal{A}$ or $\mathcal{B}$, we match Bracha's message complexity, but our protocols are constructive, whereas Bracha's is not.

6. Conclusions. In this paper we have formulated the problem of performing work efficiently in the presence of faults. We presented three work-optimal protocols to solve the problem. One sends $O(t \sqrt{t})$ messages and takes $O(n+t)$ time, another requires $O(t \log t)$ messages at the cost of significantly greater running time, and the third optimizes on time in the usual case (where there are few failures). In particular, in the failure-free case, it takes $n / t+2$ rounds and requires $2 t^{2}$ messages.

There are numerous open problems that remain. For example, it would be interesting to see if message complexity and running time could be simultaneously optimized. It would also be interesting to prove a nontrivial lower bound on the message complexity of work-optimal protocols. Finally, note that by trying to optimize effort, the sum of work done and messages sent, we implicitly assumed that one unit of work was equal to one message. In practice, we may want to weight messages and work differently. As long as the "weight" of a message is linearly related to the weight of a unit of work, then, of course, the complexity bounds for our algorithms continue 
to hold. However, if we weight things a little differently, then a completely different set of algorithms might turn out to be optimal. In general, it would be interesting to explore message/work/time tradeoffs in this model.

Acknowledgments. The authors are grateful to Vaughan Pratt for many helpful conversations, in particular for his help with the proof of Protocol $\mathcal{A}$. We also thank David Greenberg, Maurice Herlihy, and Serge Plotkin for their suggestions for improving the presentation of this work.

\section{REFERENCES}

[1] R.J. Anderson And H. Woll, Algorithms for the certified write-all problem, Siam Journal of Computing (to appear). A preliminary version, containing other results, appears in Proc. 23rd ACM Symposium on Theory of Computing, 1991, pp. 370-380 (with the title "Wait-free parallel Algorithms for the union-find problem").

[2] H. Attiya, A. BAR-Noy, AND D. Dolev, Sharing memory robustly in message-passing systems, Journal of the ACM (to appear). A preliminary version appears in Proc. 9th ACM Symposium on Principles of Distributed Computing, 1990, pp. 363-375.

[3] A. Bar-Noy And D. Dolev, A partial equivalence between shared-memory and messagepassing in an asynchronous fail-stop distributed environment, Mathematical Systems Theory, 26 (1993), pp. 21-39.

[4] G. Bracha, Unpublished manuscript, Department of Computer Science, Cornell University, July 1984.

[5] M. F. BRidgland And R. J. Watro, Fault-tolerant decision making in totally asynchronous distributed systems, in Proc. 6th ACM Symposium on Principles of Distributed Computing, 1987, pp. 52-63.

[6] J. F. Buss, P. C. Kanellakis, P. L. Ragde, And A. A. Shvartsman, Parallel algorithms with processor failures and delays, Journal of Algorithms, vol. 20, pp. 45-86, January, 1996.

[7] T. D. Chandra And S. Toueg, Unreliable failure detectors for reliable distributed systems, Journal of the ACM (to appear). A preliminary version appears in Proc. 10th ACM Symposium on Distributed Computing, 1991, pp. 325-340.

[8] R. De Prisco, A. Mayer, And M. Yung, Time-optimal message-efficient work performance in the presence of faults, in Proc. 13th ACM Symposium on Principles of Distributed Computing, 1994, pp. 161-172.

[9] C. Dwork, J. Y. Halpern, And H. R. Strong, Fault tolerant load management system and method, U.S. Patent 5,513,354, 1996.

[10] D. Dolev, R. Reischuk, and H. R. Strong, Early stopping in Byzantine Agreement, Journal of the ACM, 34 (1990), pp. 720-741.

[11] Z. Galil, A. Mayer, And M. Yung, Resolving message complexity of Byzantine agreement and beyond, in Proc. 36th IEEE Symposium on Foundations of Computer Science, 1995, pp. 724-733.

[12] D. Gelernter And D. Kaminsky, Supercomputing out of recycled garbage: preliminary experience with Piranha, in Proceedings of the ACM International Conference on Supercomputing, 1992, pp. 417-427.

[13] P. C. Kanellakis and A. A. Shvartsman, Efficient parallel algorithms can be made robust, Distributed Computing, 5 (1992), pp. 201-219.

[14] Z. Kedem, K. Palem, A. Raghunathan, and P. G. Spirakis, Combining tentative and definite executions for very fast dependable parallel computing, in Proc. 23rd ACM Symposium on Theory of Computing, 1991, pp. 381-389.

[15] Z. M. Kedem, K. V. Palem, And P. G. Spirakis, Efficient robust parallel computations, in Proc. 22nd ACM Symposium on Theory of Computing, 1990, pp. 138-148.

[16] C. U. Martel, A. Park, and R. Subramonian, Work optimal asynchronous algorithms for shared memory parallel Computers, SIAM Journal on Computing, 21 (1992), pp. 10701099.

[17] J. F. Shoch And J.A. Hupp, The Worm programs-early experience with a distributed computation, Communications of the ACM, 25 (1982), pp. 95-103. 